\title{
Modelos Constructivistas \\ de Aprendizaje en \\ Programas de Formación
}

Noelia OImedo Torre, Oscar Farrerons Vidal

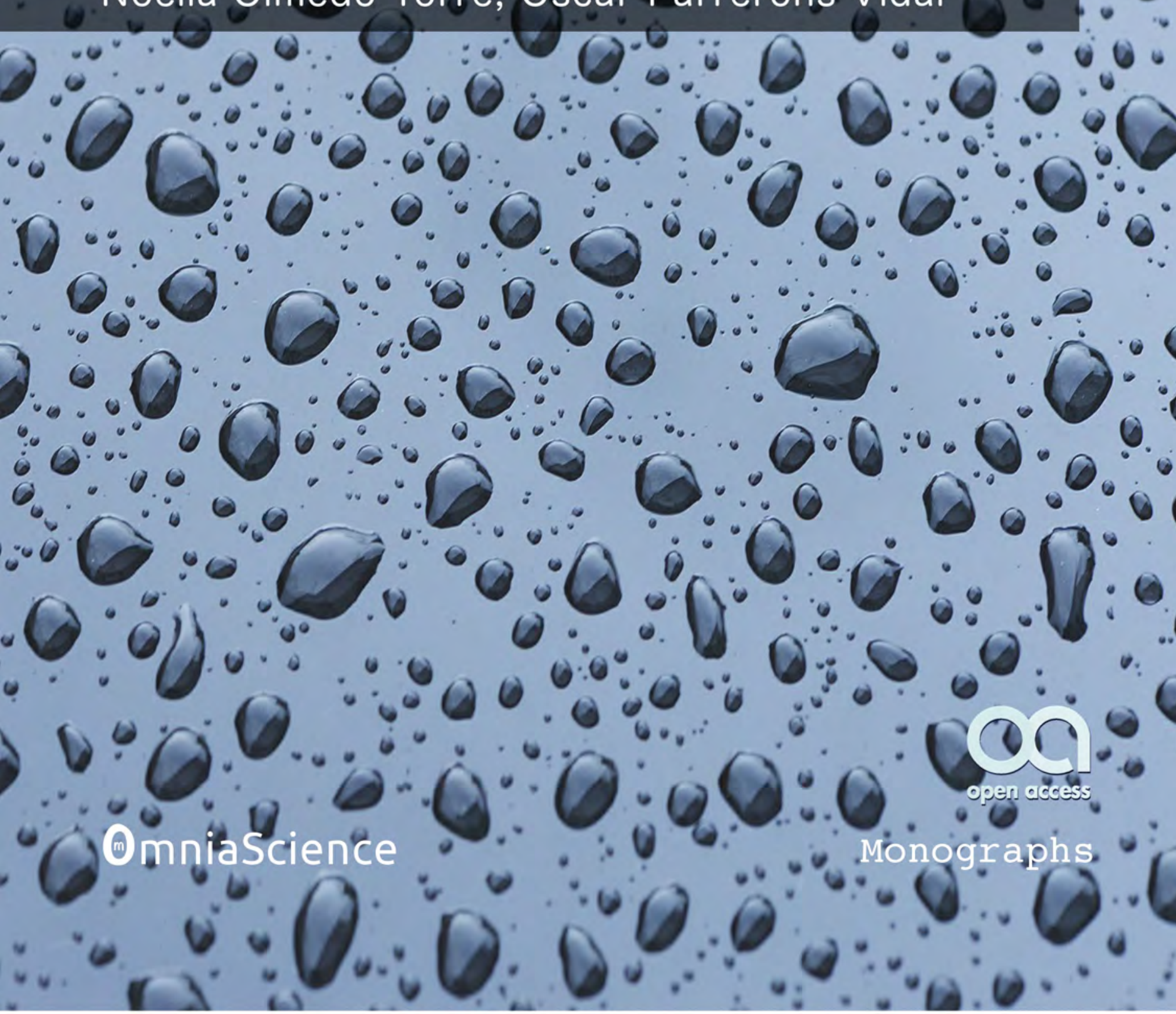


Modelos Constructivistas de Aprendizaje en

Programas de Formación

Noelia Olmedo Torre

Oscar Farrerons Vidal 


\section{Open Access Support}

$\mathrm{Si}$ encuentra este libro interesante le agradeceríamos que diera soporte a sus autores y a OmniaScience para continuar publicando libros en Acceso Abierto.

Puede realizar su contribución en el siguiente enlace: https://doi.org/10.3926/oms.367

Modelos constructivistas de aprendizaje en programas de formación

Autores:

Noelia Olmedo Torre, Oscar Farrerons Vidal

Universitat Politècnica de Catalunya, España

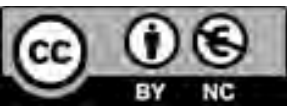

ISBN: 978-84-946352-1-2

DOI: https://doi.org/10.3926/oms.367

(C) OmniaScience (Omnia Publisher SL) 2017

(C) Cover design: OmniaScience

(C) Cover images: boristrost - Pixabay

OmniaScience no se hace responsable de la información contenida en este libro y no aceptará ninguna responsabilidad legal por los errores u omisiones que puedan existir. 


\section{ÍNDICE}

Índice de figuras $\ldots \ldots \ldots \ldots \ldots \ldots \ldots \ldots \ldots \ldots \ldots, \mathrm{V}$

Índice de tablas $\ldots \ldots \ldots \ldots \ldots \ldots \ldots \ldots \ldots \ldots \ldots \ldots \ldots \ldots$ VII

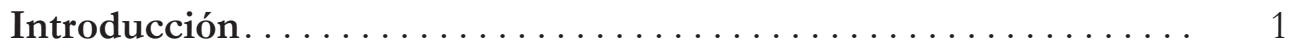

Capítulo 1. Un acercamiento al constructivismo ........... 3

1.1. El constructivismo de Piaget $\ldots \ldots \ldots \ldots \ldots \ldots \ldots \ldots \ldots$. 9

1.2. El constructivismo y otros autores.................. 11

1.3. El enfoque constructivista de Jonassen . . . . . . . . . . . 14

1.4. Entornos constructivistas de aprendizaje ............. 15

1.5. Objetos de aprendizaje (Learning Objects) ............... 19

Capítulo 2. Teorías constructivistas de Diseño Instruccional (IDT) . . 23

2.1. Aprender haciendo (Learning by Doing)................. 25

2.2. Múltiples acercamientos a la comprensión (Multiple

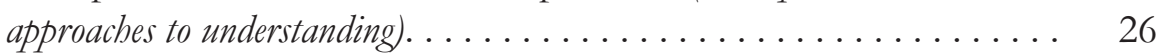

2.3. Teoría del compromiso (Engagement Theory)............. 28

2.4. Teoría de la transacción instruccional (Instructional Transaction Theory) ........................... 29

2.5. Teoría de la presentación de componentes (Component

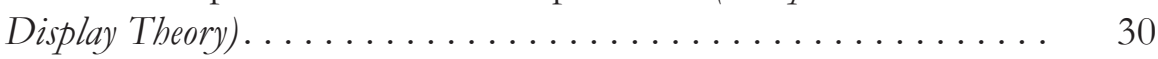

2.6. Formación de adultos ...................... 32

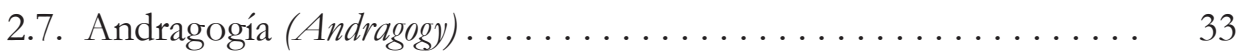

2.8. Teoría de la instrucción anclada (Anchored Instruction) ........ 36 
2.9. Modelo GOMS (Goals, Operators, Methods, and Selection rules) ..... 36

2.10. Minimalismo ............................... 38

2.11. Aprendizaje situado (Situated Learning) ................ 40

Capítulo 3. El nuevo paradigma docente $\ldots \ldots \ldots \ldots \ldots \ldots \ldots \ldots 41$

3.1. Diferentes modelos docentes . . . . . . . . . . . . . . . . 41

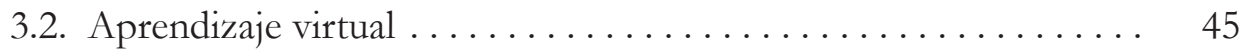

3.3. Aprendizaje semipresencial. ...................... 49

3.4. Modelos de educación a distancia $\ldots \ldots \ldots \ldots \ldots \ldots \ldots \ldots . \ldots \ldots$

3.5. Nuevos escenarios universitarios virtuales. ........... 52

Capítulo 4. Conclusiones ......................... 55

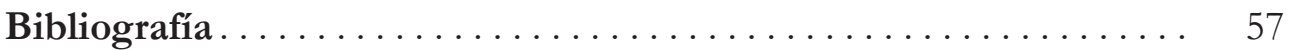

Autores..................................... 71 


\section{ÍNDICE DE FIGURAS}

Figura 1. Características que favorecen el aprendizaje en entornos constructivistas................... 15

Figura 2. Modelo docente clásico ..................... 42

Figura 3. Nuevo paradigma docente $\ldots \ldots \ldots \ldots \ldots \ldots \ldots \ldots \ldots$

Figura 4. Características de un aula virtual. ............... 46

Figura 5. Entornos de aprendizaje virtual ................ 47 



\section{ÍNDICE DE TABLAS}

Tabla 1. Modelos de aprendizaje, teorías relacionadas y proponente principal $\ldots \ldots \ldots \ldots \ldots \ldots \ldots \ldots \ldots$

Tabla 2. Resumen de las propuestas de Knowles sobre las bases teóricas que sustentan el proceso educativo de los adultos referidas a los elementos del proceso en los «Modelos Pedagógico y Andragógico».. ....................... 



\section{INTRODUCCIÓN}

«El aprendizaje de verdad solo sucede cuando alguien está realmente interesado en averiguar por qué algo ha llamado su atención o le ha dejado perplejo. Se trata de "provocar" algo dentro de cada persona, de llamarla y llegar dentro de ella».

Todos los expertos coinciden que detrás de cualquier actividad de aprendizaje se encuentra un modelo de aprendizaje. Para facilitar al lector la comprensión de estos modelos, revisaremos una de las teorías más influyentes en la educación, tanto en lo que respecta a las elaboraciones teóricas como en la propia práctica pedagógica: la teoría constructivista. Esta teoría nos allanará el camino para describir modelos que parten del contexto constructivista. Estos modelos nos serán útiles para diseñar estrategias de instrucción y técnicas que faciliten el aprendizaje, así como los fundamentos para seleccionarlos de manera eficaz.

En este libro queremos mostrar a los lectores distintos modelos de formación que serán discutidos en torno a algunas ideas fundamentales:

1. El alumno es el responsable de su propio aprendizaje; este es un proceso activo de construcción más que de adquisición de conocimiento.

2. Los contenidos elaborados son el motor y el apoyo de la actividad constructivista del alumno más que de comunicar conocimientos.

3. El docente debe crear condiciones óptimas para el despliegue de actividades constructivistas. 
2 MODELOS CONSTRUCTIVISTAS DE APRENDIZAJE EN PROGRAMAS DE FORMACIÓN

El constructivismo es parte fundamental en el proceso de aprendizaje de los estudiantes de todos los ámbitos formativos y como veremos en este trabajo el procedimiento de aprendizaje es más importante que los propios contenidos. Los modelos constructivistas nos ayudarán a diseñar estrategias de instrucción y técnicas que faciliten el aprendizaje, así como los fundamentos para seleccionarlos de manera eficaz. 


\section{1}

\section{UN ACERCAMIENTO AL CONSTRUCTIVISMO}

Si bien una teoría proporciona una explicación general a observaciones científicas, un modelo es una figura mental que nos ayuda a entender las cosas que no podemos ver o explicar directamente.

Schunk (1999) definió que las numerosas teorías de aprendizaje se pueden agrupar en tres grandes modelos:

- Conductistas: Este modelo se centra en que las personas aprenden una conducta o comportamiento del mundo externo. Aprender se considera como la formación de asociaciones entre estímulos y respuestas. Dicho de otra manera, el conductivismo se basa en los cambios observables en la conducta de un sujeto y se enfoca hacia la repetición de patrones de conducta.

- Cognoscitivas: Los modelos cognoscitivos se centran en el proceso de aprendizaje que origina el cambio de conducta. Se considera que el aprendizaje de nuevos conocimientos es lo que hace posible esos cambios; estos son observados para usarse como indicadores para entender lo que pasa en la mente del que aprende. Al igual que en el modelo anterior, se trata de construir mapas de realidad (Mergel, 2005). 
- Constructivistas: Se basa en que cada persona construye su propia perspectiva del mundo que lo rodea a través de sus propias experiencias y esquemas mentales desarrollados.

Se considera al modelo constructivista como el más influyente en el ámbito de la didáctica de las ciencias. Éste se reduce a cuatro submodelos principales (Marín-Martínez, Solano-Martínez \& Jiménez-Gómez, 1999; Marín-Martínez, 2003):

- Piagetiano: Se aplican en el ámbito de la enseñanza las diferentes partes del entramado teórico de las aportaciones de Piaget.

- Humano: Se fundamenta en la propuesta de aprendizaje significativo de Ausubel (1982). A sus seguidores se deben los mapas conceptuales o V de Gowin (Aguirre, Meza \& Lucero, 2005).

- Social: Denominado inicialmente movimiento de las concepciones alternativas. Este modelo utiliza mensajes sencillos como las concepciones específicas de los alumnos sobre los contenidos de enseñanza (Driver \& Easley, 1978).

- Radical: Promueve a comienzos de la década de los noventa ciertas actividades en el ámbito de la enseñanza más vinculada a las especulaciones y confrontación filosófica que a abordar cuestiones del aula (Marín-Martínez et al., 1999)

A modo de resumen, la Tabla 1 expone los tres modelos explicados, las distintas teorías relacionadas y el proponente principal de cada una de ellas (Jáuregui, 2002):

Los modelos constructivistas estudian el proceso de aprendizaje que provoca el cambio de conducta que, a diferencia de los modelos cognoscitivos, ponen énfasis en lo social, la cultura, el humanismo y la subjetividad como factores críticos (Jáuregui, 2002).

Tal y como Kemp y Smellie (1989) describieron, existen generalizaciones en las distintas teorías del aprendizaje, como pueden ser: 


\begin{tabular}{|c|c|c|}
\hline ClasificaCión & TEOrías RELACIONADAS & Proponente PRINCIPAL \\
\hline \multicolumn{3}{|c|}{ Modelos conductistas } \\
\hline & $\begin{array}{l}\text { Conexión entre estímulo y } \\
\text { respuesta: Ley del efecto y } \\
\text { ejercicio }\end{array}$ & Thorndike (1906) \\
\hline & Condicionamiento clásico & Pavolv (1927) \\
\hline & $\begin{array}{l}\text { Condicionamiento sin } \\
\text { reforzamiento }\end{array}$ & Watson (1916) \\
\hline & $\begin{array}{l}\text { Condicionamiento a través } \\
\text { de reforzamiento }\end{array}$ & Hull (1920) \\
\hline & Condicionamiento operante & Skinner (1938) \\
\hline \multicolumn{3}{|c|}{ Modelos cognoscitivos } \\
\hline \multirow{4}{*}{$\begin{array}{l}\text { Procesamiento } \\
\text { de la información }\end{array}$} & Corriente de Gestalt & Ehrenfels (1890) \\
\hline & Memoria a corto plazo & Miller (1956) \\
\hline & Aprendizaje multicanal & Hartman (1961) \\
\hline & Aprendizaje dual & Paivio (1986) \\
\hline \multirow{2}{*}{$\begin{array}{l}\text { Cognoscitivo } \\
\text { social }\end{array}$} & Aprendizaje por expectativas & Tolman, Hall y Bretnall (1932) \\
\hline & Aprendizaje social & Bandura, Adams y Beyer (1977) \\
\hline \multirow{5}{*}{$\begin{array}{l}\text { Cognoscitivo de } \\
\text { tareas complejas }\end{array}$} & Experimentación & Dewey (1916) \\
\hline & Teoría del cambio social & Lewin y Gullickson (1948) \\
\hline & Crecimiento cognoscitivo & Bruner (1966) \\
\hline & Aprendizaje significativo & Ausubel (1963) \\
\hline & Resolver problemas & Simon (1916) \\
\hline \multicolumn{3}{|c|}{ Modelos constructivistas } \\
\hline & Desarrollo cognoscitivo. & Piaget (1954) \\
\hline & Aprendizaje de acción. & Revans (1998) \\
\hline & $\begin{array}{l}\text { Pensamiento y lenguaje: El } \\
\text { medio social es crucial } \\
\text { para el aprendizaje. }\end{array}$ & Vygotsky (1978) \\
\hline & Teoría de acción. & Argirys y Schön (1974) \\
\hline & Reflexión en acción. & Schön (1987) \\
\hline
\end{tabular}

Tabla 1. Modelos de aprendizaje, teorías relacionadas y proponente principal. 


\section{- Motivación:}

El que aprende ha de sentirse motivado, interesado y sentir la necesidad de aprender. También los medios instructivos y los soportes de la enseñanza deben ser motivadores en cuanto al tema: presentación, tipo de letra, legibilidad, etc.

\section{- Objetivos de aprendizaje:}

Al sujeto de aprendizaje y más si es adulto, le interesa saber desde el principio qué va a aprender; por esta razón cualquier soporte audiovisual o actuación docente debería anticipar los objetivos que espera alcanzar.

- Ritmos y diferencias individuales:

La figura del individuo en relación con sus capacidades, actitudes y habilidades debe estar presente a la hora de abordar las situaciones de enseñanzaaprendizaje.

\section{- Conocimiento de los destinatarios y diseño de la acción:}

Se debe establecer un diagnóstico de los sujetos a los que destinamos nuestra acción, para ello es necesario conocer al grupo: sus intereses, el nivel de conocimientos, sus necesidades, las finalidades, etc. Tales informaciones nos permitirán enmarcar nuestras acciones y preparar la documentación, las actividades, los materiales de trabajo, etc.

\section{- La organización del contenido:}

Se deben seleccionar los contenidos; tienen que ser relevantes, significativos y al nivel de los destinatarios. Estos deben estructurarse en unidades o bloques de sentido íntegro. La parte más compleja consiste en secuenciar los contenidos, unidades y bloques entre ellos; y secuenciar los contenidos dentro de ellos, confiriéndoles sentido, direccionalidad y uniformidad. 
- Participación:

El individuo ha de participar y comprometerse en el desarrollo de la acción de aprender. La enseñanza debe pretender involucrar al máximo al alumno y hacerle participar en ese proceso. La participación debe ser frecuente y de calidad.

Las características fundamentales de un modelo didáctico constructivista se resumen a continuación (Jiménez, 1991):

La visión constructivista:

- Fundamento psicológico: Aprendizaje significativo relacionado con los conocimientos previos.

- Fundamento epistemológico: Interpretación de la realidad a través de modelos. La realidad se analiza a través de una teoría.

- Fundamentos empíricos: Ideas previas de los alumnos que mantienen a pesar de la instrucción.

Principios:

- Aprender reconstruyendo los conocimientos.

- Los contenidos y los procesos son complementarios.

- Enseñar es favorecer el aprendizaje planificando y organizando.

Sintaxis:

- Partir de las ideas del alumnado.

- Explorar, reestructurar y aplicar nuevas ideas. 
- Propiciar el cambio conceptual.

- El currículum es un programa de actividades.

- Crear situaciones de aprendizaje en las que los estudiantes construyen sus propios significados.

\section{Sistema social:}

- El profesorado es investigador, intenta seleccionar los problemas de aprendizaje.

- Flexible. Activa la participación de los estudiantes.

- Se favorece el trabajo en pequeños grupos.

- Se evalúan conceptos, habilidades, procedimientos y la capacidad de resolver nuevos problemas.

- Control de los estudiantes del propio aprendizaje.

\section{Sistema de apoyo:}

- Recursos variados.

- Formación del profesorado (integración de los contenidos disciplinares, psicopedagógicos y de didáctica de las ciencias).

- Materiales, libros, guiones de trabajo.

- Diálogo y aprendizaje cooperativo.

Consideremos ahora algunos principios de aprendizaje que se asocian a la concepción constructivista del aprendizaje y la enseñanza:

- El aprendizaje implica un proceso constructivo interno, por lo que se considera subjetivo y personal. 
- El aprendizaje se facilita gracias a la mediación con los otros, lo que conlleva a decir que el aprendizaje es social y cooperativo.

- El grado de aprendizaje depende del nivel de desarrollo cognitivo, emocional y social, así como de la naturaleza y estructuras de conocimiento.

- El inicio del aprendizaje son los conocimientos y experiencias previos que tenga el sujeto.

- El aprendizaje se facilita mediante apoyos que logren conducir a la construcción de puentes cognitivos entre lo nuevo y lo ya conocido.

\subsection{El constructivismo de Piaget}

Según Piaget, la teoría constructivista se basa en que el conocimiento es el resultado de un proceso de construcción en el que la persona participa activamente. Piaget da mayor importancia al proceso interno de razonamiento que a la manipulación externa. Por ende, se reconoce la influencia ejercida tanto por los sentidos como por la razón.

El aprendizaje es un proceso de construcción interno, activo e individual. El desarrollo cognitivo supone la adquisición sucesiva de estructuras mentales más organizadas y complejas sin una excesiva intervención del profesor.

Para Piaget el aprendizaje es un proceso de construcción activa que no depende solo de la simulación externa, sino que está determinado por el grado de desarrollo interno. Las relaciones sociales favorecen el aprendizaje y la experiencia física es una condición necesaria para que este se produzca (Sagales, 2001).

Numerosos autores (Carretero, 1993, 1998; Coll, 1997, 1998; Gómez-Granell \& Coll, 1994; Resnick, 1999) consideran que los aportes de Piaget se enmarcan en lo que se denomina «perspectiva o concepción constructivista». Piaget quiso demostrar que el aprendizaje no se produce por acumulación de conocimiento, sino porque existen mecanismos internos de asimilación y acomodación. Para la asimilación es necesario el establecimiento de relaciones entre los conocimientos previos y los nuevos, donde la acomodación se consigue reestructurando el propio conocimiento. 
Piaget establece la diferencia entre el aprendizaje en sentido restringido, cuando se adquieren nuevos conocimientos a partir de la experiencia y el aprendizaje en sentido amplio, en este caso se refiere a la adquisición de técnicas o instrumentos de conocimiento.

Para Piaget el constructivismo significa que el sujeto, mediante su actividad física y mental, va avanzando en el progreso intelectual del aprendizaje pues el conocimiento para el autor no está en los objetos ni previamente en nosotros; es el resultado de un proceso de construcción en el que participa de forma activa la persona.

En esta teoría se da más importancia al proceso interno de razonar que a la manipulación externa en la construcción del conocimiento, aunque se reconoce la mutua influencia que existe entre la experiencia de los sentidos y de la razón. Es decir, la persona va construyendo su propio conocimiento (Bandura, 2005).

La construcción de conocimientos evoluciona desde las teorías de Piaget que parte de un proceso fundamentalmente individual con un papel secundario del profesor, a una construcción social donde la interacción con los demás a través del lenguaje es muy importante.

El pensamiento de Piaget en relación con el aprendizaje se resume del siguiente modo (Piaget, 1981):

1. Es un proceso de construcción activa por parte del sujeto, el cual mediante su actividad física y mental determina sus reacciones ante la estimulación ambiental.

2. No depende solo de la estimulación externa, también está determinado por el nivel de desarrollo del sujeto.

3. Es un proceso de reorganización cognitiva.

4. Las relaciones sociales favorecen el aprendizaje siempre que produzca contradicciones que obliguen al sujeto a reestructurar sus conocimientos.

5. La experiencia física es una condición necesaria para que se produzca el aprendizaje, pero no es suficiente; se necesita además la actividad mental. 


\subsection{E1 constructivismo y otros autores}

La definición de Duffy y Jonassen $(1991,1992)$ sobre el constructivismo es que el significado del mundo es impuesto por la persona y, por lo tanto, habrá muchas maneras de estructurar el mundo y muchos significados para cada evento. Sostiene además que cada estudiante construye su propio y único significado de los eventos que aprende.

Actualmente los puntos de vista que se ubican bajo el término «constructivismo» coinciden en dos aspectos fundamentales:

1. El aprendizaje es un proceso activo de construcción más que de adquisición de conocimiento.

2. La instrucción es un proceso de apoyo a esa construcción más que comunicar conocimientos (Duffy \& Cunningham, 1996).

Seymour Papert (2002) desarrolló la que denomina «Teoría Construccionista del Aprendizaje», que defiende que el aprendizaje se produce en mejores condiciones cuando los alumnos se involucran en la creación o construcción de algo que puede ser compartido (Papert \& Harel, 2002).

Esto nos llevaría a utilizar un modelo de trabajo en el que se establezca un proceso de interiorización de elementos externos y exteriorización de elementos internos por parte de alumnos y profesores, imprimiendo el sello personal en la creación.

Las herramientas tecnológicas facilitan a los niños la adquisición de habilidades que les permiten explorar y desarrollar su capacidad para inventar, construir y diseñar proyectos. Con el desarrollo de estos proyectos los niños adquirirán unos conocimientos que además de almacenarse en sus mentes también podrán ser puestos en práctica de forma efectiva.

César Coll dice que la incorporación de conocimiento se producirá si se suministra una ayuda específica a través de la participación del alumno en actividades intencionales, planificadas y sistemáticas que logren propiciar una actividad mental constructivista. 
La concepción constructivista de Coll (1990) organiza tres ideas fundamentales:

1. El alumno es responsable de su propio proceso de aprendizaje.

2. La actividad mental constructivista del alumno se aplica a contenidos que poseen ya un grado considerable de elaboración. No es necesario que el alumno, en todo momento, descubra o invente el conocimiento escolar.

3. La función del docente es engarzar los procesos de construcción del alumno con el saber colectivo culturalmente organizado. La función del profesor no se limita a crear condiciones óptimas para que el alumno desarrolle una actividad mental constructiva, lo que debe hacer es orientar y guiar explícitamente la actividad.

A la par de los estudios de Piaget, Vygotsky comenzó a estudiar el impacto del medio y de las personas que rodean al niño en el proceso de aprendizaje y desarrolló la teoría del «Origen social de la mente» (Wertsch, 1988) y aportó el concepto de «Zona de desarrollo próximo». Según Vygotsky, cada alumno es capaz de aprender una serie de aspectos que tienen que ver con su nivel de desarrollo, pero existen otros fuera de su alcance que pueden ser asimilados con la ayuda de un adulto o de iguales más aventajados. Este tramo entre lo que el alumno puede aprender por sí mismo y lo que puede aprender con ayuda es lo que denomina «Zona de desarrollo próximo» (Martín, 1992).

Vygotsky dice que se aprende en interacción con los demás y se produce el desarrollo cuando internamente se controla el proceso, integrando las nuevas competencias a la estructura cognitiva.

La diferencia sustancial entre las ideas de Piaget y Vygotsky consiste en el mayor énfasis que pone el segundo en la influencia del aprendizaje en el desarrollo. Para Vygotsky el aprendizaje contribuye al desarrollo, es decir, es capaz de tirar de él; esta consideración asigna al profesorado y a la escuela un papel relevante al conceder a la acción didáctica la posibilidad de influir en el mayor desarrollo cognitivo del alumno (Nieda \& Macedo, 2005).

En 1963 Ausubel publica «Psicología del aprendizaje verbal significativo». Años más tarde, sus ideas fueron incorporadas por Joseph Novak a sus programas de investigación. 
Ausubel acuña el concepto de «aprendizaje significativo» para distinguirlo del repetitivo o memorístico y señala el papel que juegan los conocimientos previos del alumno en la adquisición de nuevos contenidos ${ }^{1}$.

Este autor estima que aprender significa comprender y para ello es condición indispensable tener en cuenta lo que el alumno ya sabe sobre aquello que se le quiere enseñar y propone la necesidad de diseñar para la acción docente lo que llama «organizadores previos», una especie de puentes cognitivos o anclajes, a partir de los cuales los alumnos puedan establecer relaciones significativas con los nuevos contenidos.

Defiende un modelo didáctico de transmisión-recepción significativo que supere las deficiencias del modelo tradicional al tener en cuenta el punto de partida de los estudiantes y la estructura y jerarquía de los conceptos.

Ausubel considera que lo que realmente condiciona el aprendizaje es la cantidad y calidad de los conceptos relevantes y las estructuras proposicionales que posee el alumno.

Para Ausubel y Novak, lo fundamental, por lo tanto, es conocer las ideas previas de los alumnos. Proponen para ello la técnica de los mapas conceptuales (Moreira y Novak, 1988) que son capaces de detectar las relaciones que los alumnos establecen entre los conceptos.

Ausubel definió tres condiciones básicas para que se produzca el aprendizaje significativo:

1. Que los materiales de enseñanza estén estructurados lógicamente con una jerarquía conceptual, situándose en la parte superior los más generales, inclusivos y poco diferenciados.

2. Que se organice la enseñanza respetando la estructura psicológica del alumno, es decir, sus conocimientos previos y sus estilos de aprendizaje.

3. Que los alumnos estén motivados para aprender.

${ }^{1}$ La importancia de los conocimientos previos había sido ya anteriormente sugerida por Bartlett (1932) y Kelly (1955), pero adquiere mayor protagonismo al producirse gran coincidencia en las investigaciones durante los años 70’ (Ausubel, 1963, Viennot, 1976 y Novak, 1997). 


\subsection{El enfoque constructivista de Jonassen}

Según David Jonassen (1991) el objetivo del aprendizaje se centra en proveer múltiples perspectivas de lo que nos rodea para lograr que la persona que aprende construya su propio entendimiento.

Jonassen (2005) comenta que la percepción más equivocada del constructivismo es la de creer que cada quien construye una realidad única y que la realidad solo existe en la mente del que la conoce, lo cual conduce a una anarquía intelectual.

Además, señala (Jonassen \& Reeves, 1996) que el hipertexto e hipermedia facilitan que la persona construya su propio conocimiento y afirma que Internet permite explorar gran cantidad de información y reflexionar desde múltiples perspectivas, creencias y visiones del mundo, lo cual proporciona que la persona construya su propio conocimiento. Además, la red y sus herramientas facilitan el aprendizaje cooperativo, lo que permite que las personas trabajen juntas y discutan, desarrollando así sus procesos cognoscitivos y construyendo sus propios conocimientos (Jonassen, Peck \& Wilson, 1999).

Duffy y Jonassen (1992) y Duffy, Lowyck \& Jonassen (1992) propone ocho características para favorecer el aprendizaje en entornos constructivistas (Figura 1):

- Activo: La actitud de los estudiantes debe ser responsable y activa para comprometerse en el proceso de aprendizaje.

- Constructivo: La integración de nuevas ideas en los conocimientos previos para construir nuevos significados.

- Colaborativo: El soporte social y la contribución de todos los miembros realzan el trabajo de los estudiantes.

- Intencional: Los estudiantes aprenden más si persiguen intencionadamente un objetivo de aprendizaje.

- Complejo: Es mejor que los estudiantes resuelvan problemas complejos y parcialmente planteados antes que problemas excesivamente simplificados. 


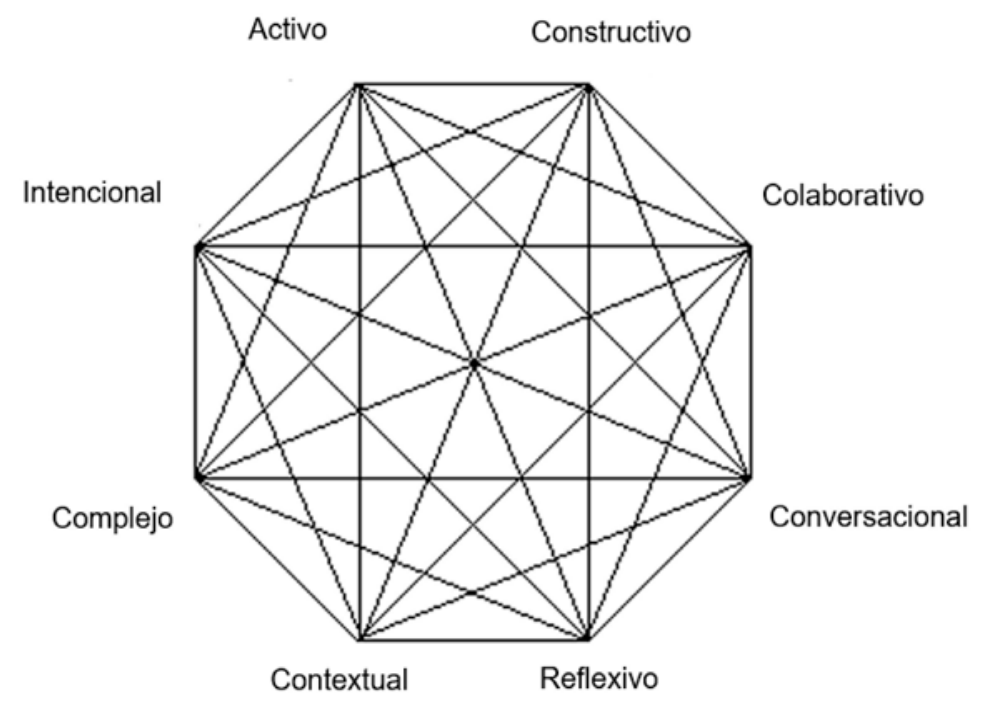

Figura 1. Características que favorecen el aprendizaje en entornos constructivistas.

- Contextual: Las actividades de aprendizaje deben estar en un contexto real o simulado. Utilizar entornos de aprendizaje basados en ejercicios sobre casos. Enseñar en la vida real proporcionando nuevos contextos para aplicar los conocimientos.

- Conversacional: Aprender es un proceso social fundamentado en el diálogo.

- Reflexivo: La tecnología permite a los estudiantes tomar decisiones en su proceso de aprendizaje. Su control en el proceso le permite aprender mejor.

\subsection{Entornos constructivistas de aprendizaje}

Según Bodner (1986), Jonassen (1991) y Duffy y Jonassen (1992), la teoría del constructivismo y el diseño de entornos de aprendizaje constructivista despierta un gran interés por las posibilidades que esta puede proveer al alumno.

Bodner (1986) dice que el modelo constructivista de conocimiento se puede resumir en la siguiente frase: «El conocimiento es construido en la mente del alumno». 
Moore, Burton y Myers (1996) analizaron estudios de diferentes autores y encontraron que algunos de ellos afirmaban que el aprendizaje a través de sistemas interactivos multimedia es igual o más efectivo que el convencional y más rápido; otros estudios determinaron la incapacidad de demostrar que el aprendizaje con tecnología multimedia era más apropiado que el aprendizaje convencional. Diferentes autores (Makkonen, 1998; Kaynama \& Keesling, 2000; Jonassen \& Reeves (1996) afirman que una aplicación multimedia se considera un elemento que facilita que la persona construya su propio conocimiento.

A su vez, Kozma (1991) menciona que la tecnología multimedia hace un paralelismo de los modelos mentales formando asociaciones entre varias ideas construyendo un significado a partir de estas relaciones.

Rouet, Levonen, Dillon y Spiro (1996) ponen de relieve la analogía existente entre la estructura de un hipertexto y los conceptos de la mente humana. En esta línea de pensamiento Vygotsky (1978) destaca que la interacción social juega un papel importante en el proceso de aprendizaje.

Reigeluth (1999) define dos tipos de interacción en el proceso de aprendizaje:

- Humana. Se consideran tres tipos:

- participante-profesor

- participante-participante

- participante-otras personas

- No bumana. Se clasifican en:

- participante-herramienta

- participante-información

- participante-entorno

- participante-otros 
En este camino y de acuerdo con Kahn y Friedman (1993) el aprendizaje constructivista se caracteriza por los siguientes principios:

1. De la instrucción a la construcción: Aprender significa transformar el conocimiento.

2. Del refuerzo al interés: Desde una perspectiva constructivista, los profesores implican al estudiante en el proyecto de aprendizaje.

3. De la obediencia a la autonomía: El profesor debe fomentar una libertad responsable.

4. De la coerción a la cooperación: A través de las relaciones entre alumnos se desarrollan los conceptos de igualdad, justicia y democracia y progresa el aprendizaje académico.

Jonassen (2000) describió algunas formas prácticas de diseñar actividades y organizar la información de acuerdo al enfoque constructivista en entornos abiertos. Este método se conoce como EAC (Entornos de Aprendizaje Constructivista) y su principal objetivo es fomentar la solución de problemas y su desarrollo conceptual.

El Modelo EAC consiste en una propuesta que parte de un problema, pregunta o proyecto como núcleo del entorno para el que se ofrecen al alumno varios sistemas de interpretación y de apoyo intelectual derivado de su alrededor. El alumno ha de resolver el problema o finalizar el proyecto o hallar la respuesta a las preguntas formuladas.

Los elementos que constituyen el modelo de Jonassen son:

a) Las fuentes de información y analogías complementarias relacionadas.

b) Las herramientas cognitivas.

c) Las herramientas de conversación / colaboración.

d) Los sistemas de apoyo social / contextual. 
El entorno debe proporcionar al alumno herramientas para apoyar las funciones necesarias para elaborar la información. Las herramientas cognitivas pueden ser herramientas informáticas cuyo propósito es abordar y facilitar tipos específicos de procedimientos cognitivos.

Estas herramientas sirven como:

- Herramientas de visualización: donde representan de una mejor manera el problema o ejercicio que se esté realizando.

- Herramientas de modelización del conocimiento: ayudan a promover en el alumno sus propios conocimientos.

- Herramientas de apoyo a la representación: sirven para consolidar esquemas preexistentes en el aprendiz mediante la automatización de los ejercicios de un nivel inferior.

- Herramientas de recopilación de la información: ayuden a reagrupar la información pertinente y necesaria para resolver un problema.

Algunas de las herramientas propuestas por Jonassen para crear EAC son Jonas$\operatorname{sen}(2000)$ :

- Herramientas de representación de problemas y ejercicios:

Se basan en un modelo mental para la comprensión de una situación. Estas herramientas proporcionan representaciones congruentes de razonamiento que permiten a los alumnos asimilar mejor la realidad.

- Herramientas para hacer modelos sobre el conocimiento estático y dinámico:

Estas herramientas permiten representaciones que orientan al estudiante en la comprensión de los fenómenos. La construcción de modelos sobre fenómenos reales permite desarrollar actividades mentales relacionadas con el pensamiento científico como: planificar, recoger datos, acceder a la información, visualizar datos, modelizar y documentar. Bases de datos, hojas de cálculo, redes semánticas, sistemas expertos y construcciones hipermedia son algunos ejemplos de estas herramientas. 
El modelo Model-it es citado como herramienta útil para el uso de las matemáticas y como modelo de simulación que permite observar los diversos valores de determinadas relaciones entre fenómenos.

- Herramientas de apoyo al rendimiento:

Son aquellas herramientas que sirven para automatizar determinados algoritmos o rutinas necesarios para ciertas actividades cognitivas. Todos los protocolos, hojas de cálculo que permitan ordenar y organizar tareas, etc. estarían entre las herramientas para ayudar a obtener rendimientos con una mejora del tiempo.

- Herramientas para recopilar información:

Son herramientas orientadas a la búsqueda de la información, como bases de datos, fuentes de información, robots de búsqueda, etc.

- Herramientas de conversación y colaboración:

Los entornos de aprendizaje soportados con plataformas tecnológicas utilizan una gran variedad de medios de comunicación que permiten la colaboración de la comunidad educativa. Los estudiantes participan activamente en: listas de distribución, correo electrónico, servicios de noticias, boletines, chats, foros, tablones, MUDs² (multi-user dimensions) y MOOs (MUDs orientados a objetos) (Londoño, 2005).

\subsection{Objetos de aprendizaje (Learning Objects)}

Los entornos constructivistas de aprendizaje (Constructivist Learning Environment), sirven de ayuda a los estudiantes mediante el uso de herramientas cognitivas para

\footnotetext{
${ }^{2}$ Los MUDs (Multi- UserDimension) pueden describirse como servidores que proporcionan un entorno de relación entre varios usuarios, estos usuarios pueden ser autómatas, programas o personas conectadas. Pueden conversar entre usuarios, manejar objetos o moverse por espacios virtuales. Los MOOS (MUDs Object Oriented) son entornos multiusuarios que crean imágenes, objetos y espacios virtuales a partir de narraciones textuales. Los usuarios son los creadores de sus mundos virtuales.
} 
realizar actividades de aprendizaje. Los objetos de aprendizaje son contenidos educativos y procedimientos que ayudan a los estudiantes a localizar y usar el contenido, pero también actividades que ayuden a las instituciones de enseñanza a rastrear el progreso del alumno, informar sobre su desempeño y facilitar una mejor interacción entre los sistemas administrativos.

David Wiley (2000) dice que los objetos de aprendizaje son cualquier recurso digital que puede ser reutilizado para soportar aprendizaje.

La Learning Technology Standards Commitee (LTSC) ha consensuado el término Learning Objects debido a la diferencia de criterios existentes para describir a estos como «cualquier entidad digital, o no digital, que puede ser usada, reutilizada o referenciada durante el aprendizaje soportado en ordenador» (Santacruz-Valencia, 2000).

Algunos ejemplos de aprendizaje donde interviene la tecnología son:

- Sistemas de formación basados en ordenadores.

- Entornos interactivos de aprendizaje.

- Sistemas de instrucción por ordenador.

- Sistemas de formación a distancia.

- Entornos colaborativos de aprendizaje.

Los estándares de los objetos de aprendizaje se centran en (Fernández, 2003):

- Interoperabilidad: definida como la posibilidad de comunicación.

- Durabilidad: definida por tener el último valor actualizado.

- Administrable: definida por la habilitación de valores para ser evaluados.

- Reutilizable: definida por el uso en diferentes situaciones contextuales.

- Accesibilidad: definida por el acceso a los contenidos por individuos con discapacidad. 
Estos estándares centran su atención en los contenidos. Su característica fundamental es la reutilización, es decir, los diseñadores desarrollan pequeños componentes de formación que se pueden utilizar en numerosas ocasiones y en diferentes contextos de aprendizaje.

Definimos algunos tipos de objetos de aprendizaje:

- Fundamental: un recurso digital único, sin combinaciones. (por ejemplo, un archivo JPEG).

- Combinación cerrada: recursos digitales combinados que forman un solo objeto a presentar (por ejemplo, JPEG con audio).

- Combinación abierta: recursos digitales que permiten la combinación dinámica por el ordenador cuando presenta el contenido (por ejemplo, la incorporación de un texto a una imagen a tiempo real).

- Generadores de presentaciones: recursos para facilitar la presentación de información, fundamentalmente objetos de aprendizaje de contenido básico y de combinación cerrada (por ejemplo, modelos de programación utilizados para la presentación de contenidos).

- Generadores de programas de formación: recursos para generar aplicaciones de formación por combinación de otros objetos de aprendizaje o presentación. Permiten evaluar las interacciones del usuario y definir estrategias instruccionales asociadas a las respuestas (por ejemplo, los Execute Instructional Transaction Shell descritos por Merrill en 1999) definidos en su Instructional Transaction Theory (IT'T) o los modelos de programación aplicados en ejercicios, prácticas o actividades interactivas. 



\section{Capítulo \\ 2}

TEORÍAS CONSTRUCTIVISTAS DE
DISEÑO INSTRUCCIONAL (IDT)

Estas teorías hacen referencia a la forma en la que se estructura el aprendizaje, es decir, los pasos a seguir, la metodología, materiales, motivaciones, etc. Las teorías de diseño instruccional (IDT) intentan dar respuestas a la implementación de métodos de instrucción eficientes y óptimos. Su objetivo es analizar el modo en que se debe realizar el diseño instruccional, por lo que tienen gran importancia en la elaboración de materiales instruccionales.

Las IDT se refieren a la búsqueda de métodos óptimos de instrucción que proporcionen los cambios deseados en los conocimientos y destrezas del alumno.

Básicamente las IDT comprenden, aunque no siempre todos a la vez, los siguientes elementos:

- Una clasificación de los objetivos de aprendizaje.

- Una prescripción de cómo descomponer los objetivos generales en específicos.

- Una descripción de las acciones instructivas específicas y como relacionarlas con los objetivos específicos. 
- Una prescripción de la secuencia de acciones instructivas definiendo una estrategia instructiva.

- Un conjunto de condiciones para las estrategias y acciones instructivas, como por ejemplo las características del alumno.

Gagne y Dick (1983) dicen que el objetivo de los diseños instruccionales es explicitar los procedimientos estructurales del proceso de instrucción, especificando el contenido y objetivo de cada una de las etapas a seguir para asegurar la máxima eficacia en el proceso enseñanza / aprendizaje ${ }^{3}$.

A su vez Glaser (1982) analiza los modelos instruccionales a través de cuatro componentes:

- La naturaleza de la ejecución competente:

Son los procesos, estructuras de los conocimientos y capacidades que conforman el objetivo de la instrucción y que caracterizan a los sujetos competentes.

- Estado inicial del aprendiそ:

Habilidades y conocimientos que posee el aprendiz y que son necesarias para la instrucción o la facilitan.

- Procesos de aprendizaje:

Análisis del proceso de aprendizaje como guía para alcanzar los objetivos propuestos.

- Evaluación:

Comprobar el efecto del programa, en qué medida el procedimiento ha sido eficaz para conseguir los objetivos previamente marcados.

\footnotetext{
${ }^{3}$ Los argumentos de esta hipótesis fueron refutados por Streibel (1991) y Winn (1990, 1993). A pesar de ello, el eje de la idea de la educación asistida por ordenador continúa basándose en los procedimientos tradicionales del proyecto instruccional.
} 
Las teorías de la enseñanza (Casas, Fíalo \& Maia, 2003) dicen que la naturaleza de la interacción entre el estudiante y la instrucción es determinante en el aprendizaje, de igual o mayor importancia que el contenido o la forma en que la información es presentada. Uno de los defensores de estas expresiones es Merrill (1994) y se basa en la idea de que todo aprendizaje resulta de la interacción entre el estudiante y el programa. Merrill introduce un nuevo término «Instructional Component» (ITT) (Merrill, 1999) que describe la teoría de la transacción instruccional.

La ITT describe la estrategia y los métodos para utilizar los objetos del conocimiento, es decir la presentación de los componentes del conocimiento, más simplificadamente: las actividades que realizan los estudiantes y las guías didácticas para su realización.

M. David Merrill autor de la «Teoría de la Presentación por Componentes» (Component Display Theory), que describiremos más adelante, propone con la ITT criterios para programar formación basada en tecnología y describe representaciones del conocimiento, estrategias de formación y prescripciones para el diseño.

Como objetivo, la ITT incluye una formación más eficaz si se consiguen adecuar las estrategias de aprendizaje a los objetivos.

\subsection{Aprender haciendo (Learning by Doing)}

Aprender haciendo (Learning by Doing) es una metodología de aprendizaje de raíz constructivista que se basa en los estudios de Roger C. Schank (1999). Este modelo está orientado a la resolución de problemas y a la integración de los conocimientos en situaciones reales. Schank dice que la gente no aprende leyendo o escuchando, sino que solo aprende haciendo (Learning by Doing). Los problemas deben ser motivadores para los alumnos. Lo primero es definir el objetivo de la actividad y posteriormente desarrollar una historia que justifica la necesidad de cumplir la misión. Se proporciona a los estudiantes un rol para participar, para ello deberá utilizar las operaciones disponibles y las ayudas. 
Schank (2001) ${ }^{4}$ sostiene que el impacto de los ordenadores en la educación no empezará a notarse hasta que no cambie el modelo educativo y cree que el verdadero reto está en cambiar el modelo de aprendizaje, dado que los ordenadores tienen el potencial para dejar de ser un medio de hacer las mismas cosas más rápido y convertirse en una forma de hacer las cosas de una manera diferente, imposible en la vida real.

\subsection{Múltiples acercamientos a la comprensión (Multiple approaches to understanding)}

La teoría de la inteligencia múltiple de Howard Gardner sugiere que existen distintas formas de inteligencia para cada individuo y éstas residen en una localización precisa de la corteza cerebral. La diferencia radica en la forma en que cada cual desarrolla cada una de esas inteligencias. Gardner, además, comparte algunas ideas comunes con otras teorías (Cronbach \& Snow, 2003) y propone estas formas primarias de inteligencia:

- Verbal o lingüistica: El individuo se comunica a través del lenguaje.

- Musical: El individuo crea, comprende y se comunica con el sentido musical.

- Lógico-matemática: Utiliza las relaciones abstractas.

- Espacio-temporal: El individuo percibe información visual o espacial y transforma esta información recreando de memoria imágenes visuales.

- Cinético-Corporal: Usa su cuerpo para crear productos o resolver problemas.

- Personal:

- Intrapersonal: Ayuda al individuo a distinguir sus propios sentimientos, construir modelos mentales apropiados y utilizar este conocimiento en la toma de sus propias decisiones (por ejemplo, la metacognición).

\footnotetext{
${ }^{4}$ Schank, R.C. es profesor de Informática, Psicología y Educación en la Northwestern University.
} 
- Interpersonal: Le permite al individuo reconocer y distinguir los estados de ánimo, intenciones, motivos y sentimientos de otras personas (por ejemplo, habilidades sociales).

- Naturista: Distingue, clasifica y utiliza las características del medio ambiente.

- Existencial: El individuo utiliza ejemplos de vida, valores, etc.

Según Gardner, la enseñanza y el aprendizaje deben centrarse en las inteligencias particulares de cada persona. Por ejemplo, si un individuo tiene inteligencias espaciales o musicales fuertes, se le debe animar a que desarrollen estas capacidades. Gardner (1989) pone el acento en el contexto cultural de las inteligencias múltiples donde cada cultura tiende a acentuar inteligencias particulares ${ }^{5}$.

Como principios fundamentales propone:

- Animar a los alumnos a usar su inteligencia preferida para aprender.

- Las actividades de formación deben buscar y encontrar las diversas formas de inteligencia del alumno.

- El aprendizaje debe medir las formas múltiples de inteligencia.

En esta teoría, entre sus características, destacan:

- Importancia de disponer de diferentes formas de presentación de contenidos que capten el interés de los estudiantes.

- Proposición de puntos de entrada o maneras de introducir los contenidos para motivar al estudiante (narrativo, cuantitativo / numérico, fundacional, existencial, estético, manipulativo y social).

- Hacer énfasis en la publicación de los trabajos de los estudiantes.

\footnotetext{
${ }^{5}$ Gardner (1989) habla sobre las capacidades espaciales de la gente de Puluwat (Islas de Caroline), que utiliza sus habilidades para navegar en canoas por el océano y también discute el equilibrio de las inteligencias personales requeridas en la sociedad japonesa.
} 


\subsection{Teoría del compromiso (Engagement Theory)}

Esta teoría (Kearsley \& Shneiderman, 1997; Shneiderman, 1994) nace en entornos de educación a distancia con soporte telemático de la experiencia de Kearsley y Shneiderman e incorpora propuestas constructivistas y las teorías del aprendizaje situado. Está orientada a la autoformación y a las teorías de aprendizaje de adultos (por ejemplo, Androgogy).

El compromiso de aprender significa que todas las actividades del estudiante implican procesos cognoscitivos activos tales como crear, solucionar problemas, razonar, tomar decisiones, evaluar, etc. La teoría del compromiso parte de la idea de comprometer a los estudiantes en actividades de aprendizaje en colaboración con otros estudiantes creando grupos de trabajo colaborativo, participando en proyectos de interés real y siendo los profesores los que orientan este trabajo.

El aprendizaje colaborativo utiliza ordenadores como una ayuda para los procesos de aprendizaje en grupo donde el grupo aprende con ayuda de una red de comunicaciones.

Las teorías sobre aprendizaje colaborativo sugieren que en el proceso de aprender los estudiantes clarifican y discuten sus problemas y por lo tanto es un facilitador de soluciones.

La colaboración aumenta la motivación de los estudiantes y despierta y sostiene el interés de aprender (Argyro, 2003). Los tres puntos básicos de esta teoría son (Kearsley \& Shneiderman, 1997):

- El compromiso dentro del grupo: el contexto de grupo anima a los estudiantes a aprender a través de equipos de colaboración.

- Se aprende sobre la base de un proyecto definido dentro del grupo: el trabajo en grupo es la forma más popular de participación. Los estudiantes aprenden a resolver los problemas propios del proyecto.

- Contribución a la sociedad: Los estudiantes aprenden dentro del contexto de sociedad. El valor de aprender es hacer una contribución a la sociedad. Por ejemplo, la organización de la comunidad, la escuela, el estado, etc. El proceso de aprendizaje podrá ayudar a los estudiantes a entender el mundo. 
Las actividades del grupo se resumen en Relate-Create-Donate (Relacionar, Crear, Contribuir):

- Relacionar: pone de manifiesto el esfuerzo de los grupos en los procesos de comunicación, planificación, gestión y en las habilidades sociales. Estas características forman parte de muchos de los perfiles profesionales actuales.

- Crear: Se trata de llevar a cabo actividades creativas. La definición de un proyecto y su gestión permite al estudiante un mayor control sobre su proceso de aprendizaje. Esta orientación del aprendizaje mediante proyectos es lo que se conoce como Problem Based Learning (PBL) (Barrows \& Tamblyn, 1980), utilizado en muchos entornos profesionales.

- Contribuir: Destaca el valor de la contribución que se produce mientras se aprende por cuanto se trata de realizar proyectos de interés real y con personas reales: Desde la misma comunidad educativa hasta las empresas, pasando por instituciones públicas de diferente naturaleza (Jacoby \& Associates, 1996).

Los recursos que el grupo utiliza para realizar los proyectos pueden ser: e-mail, foros, chat, newsletters, aulas virtuales, reuniones presenciales, videoconferencias, bases de datos, software colaborativo, audio, etc.

\subsection{Teoría de la transacción instruccional (Instructional Transaction Theory)}

Definimos «Transacción Instruccional» (IT) como el conjunto de interacciones necesarias para que un estudiante adquiera un conocimiento o una habilidad.

Un algoritmo instruccional requiere un conjunto de objetos de conocimiento relacionados de una determinada forma (estructura de conocimiento) y que contiene todo el conocimiento necesario para adquirir el objetivo de aprendizaje propuesto.

La teoría de la transacción instruccional explica que es posible desarrollar entornos de aprendizaje interactivos y de simulación, con transacciones de identificación, de ejecución de procedimientos o de interpretación que incorporan estrategias de presentación, prácticas y ayudas al aprendizaje. 
Farhad Saba (1988) define en «Sistemas Integrados de Telecomunicaciones y Transacción Instruccional» que el que aprende a distancia recibe instrucciones a través de un canal de comunicación. Un rasgo diferenciado de la educación a distancia es la transacción instruccional mediada, siendo posible por la comunicación interactiva a través de la voz, textos, datos o cualquier información que provenga de una continuidad virtual.

\subsection{Teoría de la presentación de componentes (Component Display Theory)}

La teoría de la presentación de componentes (Merrill, 1983, 1994) (Component Display Theory) describe como se interrelacionan los objetivos de aprendizaje y los modelos de presentación.

Según Merrill los objetivos de aprendizaje combinan:

- Contenidos:

- Hechos: Piezas de información con nombre propio, fecha o acontecimiento, símbolo para designar un grupo de objetos o acontecimientos.

- Conceptos: Grupos de objetos, acontecimientos o símbolos con características comunes.

- Procedimientos: Secuencia ordenada de pasos para conseguir un objetivo, proceso o producto.

- Principios: Explicaciones o predicciones que relacionan causas y efectos.

- Rendimiento o desempeño:

- Recordar: En la memoria, una información previamente guardada.

- Usar: Aplicar alguna abstracción a un caso específico.

- Encontrar: Deducir o inventar una nueva abstracción. 
Merrill clasifica cuatro modelos de presentación de la información en la instrucción, en función de su finalidad instructiva:

1. Primarios: Se usan para presentar la información de forma general. Se especifican cuatro formas de presentación primaria:

- Reglas: presentación expositiva de una generalidad.

- Ejemplos.

- Recordar.

- Practicas: preguntas sobre los ejemplos.

2. Secundarios ${ }^{6}$ : Usados para facilitar el procesamiento de la información por parte del alumno o complementar contenidos específicos.

La presentación secundaria incluye:

- Prerrequisitos.

- Objetivos.

- Ayudas.

- Mnemónicos.

- Feedback.

3. De procesos: Son instrucciones presentadas al alumno sugiriéndole cómo procesar la información que se le ofrece.

\footnotetext{
${ }^{6}$ Merrill sugiere que la formación es tanto más efectiva cuando contiene las presentaciones primarias y secundarias necesarias y dice que existe una combinación de formas de presentación que proporciona un aprendizaje más eficaz para cada objetivo de aprendizaje.
} 
4. De procedimientos: Directrices que guían al alumno respecto a cómo operar con algún tipo de equipo en el entorno instructivo.

En 1994 Merrill rescribe la «Teoría de Presentación de Componentes» (CDT) para dar una respuesta en el campo del diseño instructivo a los nuevos desarrollos en hardware y software y la generalización de su uso en el terreno educativo (Merrill, 1994).

En esta nueva teoría se crea una distinción entre dos modos de instrucción:

- Modelo tutorial: La información se presenta al alumno de manera estructurada.

- Modelo experiencial: El alumno puede interactuar directamente con los contenidos que se presentan de forma experiencial.

\subsection{Formación de adultos}

Cross $(1976,1981)$ definió en 1991 cuales deben ser las características de la formación para adultos. El modelo integra, además, otros desarrollos teóricos para el adulto, por ejemplo, el Andragogy que veremos enseguida.

El modelo de Cross tiene en cuenta dos variables:

- Características personales: Las características personales incluyen: envejecimiento, fases de vida y etapas de desarrollo.

- Características circunstanciales: Las características circunstanciales consisten en la voluntad de aprender afectados por el tiempo disponible, es decir, horarios, localización, procedimientos.

Cross definió una serie de principios que rigen la formación del adulto:

1. Los programas de formación del adulto deben capitalizar la experiencia de los participantes.

2. Los programas deben adaptarse a las limitaciones de edad de los participantes. 
3. Los adultos deben avanzar por etapas en el desarrollo personal.

4. Los adultos deben tener opción a la disponibilidad y organización de los programas de formación.

\subsection{Andragogía (Andragogy)}

La teoría de Andragogy de Malcom Knowles (1984) es una tentativa de desarrollar una teoría específicamente dirigida al aprendizaje de adultos. Malcolm Knowles afirma que la Andragogía es el arte y ciencia de ayudar a aprender a los adultos, basándose en suposiciones acerca de las diferencias entre niños y adultos (Knowles, 1968).

Knowles especifica que uno mismo debe tomar la iniciativa y la responsabilidad de las decisiones en el aprendizaje, siendo este un aspecto fundamental en los programas de formación de adultos.

Knowles hace las siguientes apreciaciones sobre el deseo de aprender de un adulto:

- Los adultos tienen necesidad de saber y, por lo tanto, necesidad de aprender.

- Hay una necesidad de los adultos de aprender experimentalmente.

- Un buen contexto de aprendizaje es la resolución de problemas.

- Los adultos aprenden mejor cuando pueden aplicar lo aprendido inmediatamente.

Para la Andragogía, la formación para adultos necesita centrarse más en el proceso y menos en el contenido que se enseña.

Las estrategias tales como estudios de casos, roles a desempeñar, simulaciones y auto evaluación son las más útiles. En este caso, el papel de los instructores es el de facilitar los recursos necesarios.

En 1980, Knowles expuso las bases teóricas que sustentan el proceso educativo de los adultos referidas a los elementos del proceso en los «Modelos Pedagógico y Andragógico» (Knowles, 1980). 
La Tabla 2 resume sus propuestas.

Manuel Castro Pereira profundizó en la elaboración de un «Modelo Curricular Andragógico» que constituye un gran esfuerzo para operacionalizar la Andragogía como ciencia y las hipótesis y principios que le dan sustento. El trabajo en referencia conforma un medio muy importante para tener acceso al currículum y su diseño de una manera diferente, flexible, innovadora y participativa que invita tanto a observar su aplicación como a evaluar los factores que coadyuvan en la superación del adulto en situación de aprendizaje (Castro-Pereira, 2004).

\begin{tabular}{|c|c|c|}
\hline Acerca de & Modelo Pedagógico & Modelo Andragógico \\
\hline \multirow{4}{*}{ Clima } & Tenso, de poca confianza. & Relajado, confiable. \\
\hline & Formal, frío, distante. & Mutuamente respetuoso. \\
\hline & Orientado por la autoridad. & Informal, cálido. \\
\hline & Competitivo, juzgador. & Colaborativo, apoyador. \\
\hline Planificación & Básicamente por el profesor. & $\begin{array}{l}\text { Mutuamente por educandos } \\
\text { y facilitador. }\end{array}$ \\
\hline $\begin{array}{l}\text { Diagnóstico de } \\
\text { necesidades }\end{array}$ & Básicamente por el profesor. & Por mutua valoración. \\
\hline Fijación de objetivos & Básicamente por el profesor. & Por negociación mutua. \\
\hline \multirow{3}{*}{$\begin{array}{l}\text { Diseño de planes de } \\
\text { aprendizaje }\end{array}$} & Planes de contenido del profesor. & Contratos de aprendizaje. \\
\hline & Unidades didácticas del curso. & Proyectos de aprendizaje. \\
\hline & Secuencia lógica. & Secuenciados por disposición \\
\hline \multirow{3}{*}{$\begin{array}{l}\text { Actividades de } \\
\text { aprendizaje }\end{array}$} & Técnicas de transmisión. & Proyectos de investigación. \\
\hline & Lecturas asignadas. & Estudios independientes. \\
\hline & & Técnicas de experiencias. \\
\hline \multirow{3}{*}{ Evaluación } & Por el profesor. & Por evidencia reunido por el \\
\hline & $\begin{array}{l}\text { Referidos a normas (por una } \\
\text { curva). }\end{array}$ & $\begin{array}{l}\text { educando, validada por sus } \\
\text { compañeros, facilitadores y } \\
\text { expertos. }\end{array}$ \\
\hline & Con notas. & Referida a criterios \\
\hline
\end{tabular}

Tabla 2. Resumen de las propuestas de Knowles sobre las bases teóricas que sustentan el proceso educativo de los adultos referidas a los elementos del proceso en los «Modelos Pedagógico y Andragógico». 
Un modelo Andragógico encuentra su dinamismo en los siguientes componentes:

- El participante adulto.

- El andragogo.

- El grupo de participantes.

- El medio ambiente.

a) Elparticipante adulto: Es el primer y principal recurso en la situación de aprendizaje. Apoyándose en sus conocimientos y experiencias anteriores, el participante no hace más que continuar la explotación y/o descubrimiento de sus talentos y capacidades.

b) El andragogo: Es una persona reconocida como competente ya sea en el campo del aprendizaje a realizar, o cómo se puede realizar, o ambos a la vez. Como persona de referencia y/o persona experta, el andragogo puede y debe desempeñar variados roles, tales como: consultor, transmisor de informaciones, facilitador, agente de cambio, agente de relación, tutor, etc.

c) El grupo: Los adultos reunidos en grupos de participantes, constituyen un conjunto de recursos debido a sus experiencias anteriores y de su voluntad para aprender. De esta manera, cada uno de los miembros del grupo se convierte en agente de aprendizaje ya sea en lo referente al contenido o al proceso.

d) El medio ambiente: Es posible distinguir tres tipos de medio ambiente. El primero comprende el medio ambiente inmediato, creado para realizar el aprendizaje, es decir, la actividad educativa. El segundo se relaciona con el organismo educativo que facilita los recursos y los servicios humanos y materiales. El tercer tipo comprende a las instituciones y a las agrupaciones sociales.

Finalmente, Castro Pereira llegó a la siguiente conclusión: «La Andragogía es una de las ciencias de la educación que tiene por finalidad facilitar los procesos de aprendizaje en el adulto a lo largo de toda su vida.» 


\subsection{Teoría de la instrucción anclada (Anchored Instruction)}

La instrucción anclada, paradigma importante del aprendizaje basado en la tecnología fue desarrollada por el «Cognition \& Technology Group at Vanderbilt» (CTGV) y a John Bransford se le atribuye el enunciado y las principales aportaciones de esta teoría (Bransford, Sherwood, Hasselbring, Kinzer \& Williams, 1990).

La teoría de la instrucción anclada se basa en el desarrollo de herramientas interactivas. Estas herramientas animan a estudiantes y profesores a plantear y solucionaran problemas complejos y realistas. Las secuencias de vídeo sirven como «anclas» (macro contextos) para toda la formación. Se trata de historias que pretenden captar el interés y que deben ser exploradas por estudiantes y profesores.

El CTGV explica que en el diseño de estas anclas se pretende ser diferente del diseño de los sistemas interactivos típicos utilizados en la educación. El uso de la tecnología interactiva multimedia permite a los estudiantes explorar fácilmente el contenido. Esta teoría se relaciona con el «aprendizaje situado» comentada más adelante.

\subsection{Modelo GOMS (Goals, Operators, Methods, and Selection rules)}

GOMS (Goals, Operators, Methods, and Selection rules) es un método que permite describir una tarea y la manera en que un usuario deberá realizarla en lo que respecta a objetivos, operadores, métodos y reglas de selección.

GOMS es una teoría sobre habilidades cognitivas relacionadas con las tareas con ordenadores. Se basa en los tipos de memoria implicados: sensorial, memoria de trabajo y memoria a largo plazo en los procesos cognitivos.

Card, Moran y Newell (1983) propusieron la formulación original de GOMS y crearon una versión simplificada, el modelo de pulsaciones de teclas KLM (Keystroke-Level Moded).

John E. Bonnie desarrolló una versión de actividades paralelas, CPM-GOMS y David Kieras (1988) una versión más rigurosa, NGOMSL (Natural GOMS Language). 
Todas éstas técnicas se basan en el mismo concepto del GOMS.

Según el modelo GOMS, la estructura cognoscitiva consta de cuatro componentes:

- Un sistema de objetivos o metas.

- Un sistema de operadores.

- Un sistema de los métodos para alcanzar las metas.

- Un sistema de reglas de selección para elegir el método más competente.

Para una tarea dada, se puede construir una estructura particular de GOMS y utilizarla para predecir el tiempo requerido para terminar la tarea. Además, el modelo se puede utilizar para identificar y predecir los efectos en los errores de funcionamiento de la tarea.

GOMS está pensado también como metodología de diseño de un sistema. Esto permite que los diseñadores de una interfaz prueben los diseños utilizados. El modelo GOMS describe los métodos necesarios para llevar a cabo los objetivos que se desean. Estos métodos se componen de pasos que el usuario ejecuta. Cuando existe más de un método disponible para cumplir un objetivo las reglas de selección permitirán decidir cuál será el método adecuado para cada situación.

Dicho de otra manera, el modelo GOMS está compuesto de métodos para adquirir objetivos específicos. Estos métodos están compuestos de pasos específicos que realiza un usuario en un tiempo determinado de ejecución. Si un objetivo se puede conseguir por más de un método las reglas de selección se utilizan para elegir el método adecuado.

Este modelo está relacionado con el modelo minimalista de Carrol que veremos en el próximo apartado.

Describimos una serie de principios del modelo GOMS:

1. Para mejorar el funcionamiento de una habilidad cognoscitiva se debe eliminar a operadores innecesarios del método utilizado para hacer la tarea o utilizar otros métodos. 
2. Los operadores implicados en habilidades cognoscitivas son altamente específicos para los métodos usados en una tarea dada.

3. El funcionamiento de la tarea puede ser mejorado proporcionando un sistema de métodos del error-recuperación.

\subsection{Minimalismo}

La teoría del minimalismo de Carroll (1998) elaborada bajo la influencia del constructivismo de Piaget) está pensada esencialmente para la formación de usuarios a través de ordenadores. Esta teoría piensa que la instrucción debe ser breve y debe permitir al usuario su propia exploración.

El minimalismo está fuertemente centrado en el usuario, hace explícitos sus objetivos e implica al alumno en tareas reales, reduce la extensión de los materiales de adiestramiento y apoya explícitamente el reconocimiento y la corrección de errores.

Sus objetivos son:

- Mantener la motivación.

- Promover el aprendizaje activo.

- Hacer seguro el entorno de aprendizaje en el sentido de permitir al usuario que experimente con el programa sin que se sienta frustrado cuando comete errores.

Esta teoría sugiere que:

- Todas las tareas que los alumnos aprenden son actividades significativas y autónomas.

- Se deben realizar proyectos realistas y lo más rápidamente posible.

- La planificación de la formación debe potenciar el propio razonamiento de los estudiantes y su improvisación aumentando las actividades activas de aprendizaje. 
- Los materiales y actividades de enseñanza deben prever el error y la recuperación.

- Debe existir una total relación entre el sistema de formación y el sistema real.

La teoría del minimalismo está pensada para ser construida sobre la experiencia del principiante. Generalmente los usuarios inexpertos exploran las funciones del programa mediante el sistema ensayo-error.

El modelo minimalista asume dos importantes principios de la psicología cognitiva:

1. El constructivismo: los usuarios construyen sus propios modelos mentales combinando sus anteriores experiencias con la nueva información procedente de la pantalla del ordenador y de la documentación.

2. El aprendizaje activo: los usuarios aprenden mejor cuando se implican de forma activa, cuando hacen algo por sí mismos, no solo seguir instrucciones a la manera de un guion (Charney, Reder \& Wells, 1988).

Carroll elaboró una guía para la elaboración de documentación que integra el constructivismo y el aprendizaje activo (Tejada, 1999):

- Dar oportunidades a los usuarios para que formen sus propios modelos mentales. Invitarlos a explorar y descubrir por si mismos en lugar de dirigirlos siempre paso a paso a través de un ejemplo (Van der Meij, 1995).

- No decir a los usuarios todo acerca del programa, omitir lo que ya saben o pueden inferir y omitir en la documentación lo que pueden fácilmente ver en la pantalla del ordenador (Carroll, 1998).

- Asumir que los usuarios cometerán errores, porque de hecho se producen en cualquier situación de aprendizaje. Hay una necesidad de prevenir errores, pero también de averiguar qué tipos de errores son más probables que cometan los usuarios en un momento dado del adiestramiento y ayudarles a que los reconozcan y corrijan (Carroll, 1987; Lazonder \& Van der Meij, 1995; Hackos \& Redish, 1998). 
Carroll piensa que la formación desarrollada en base a otras teorías educacionales (Gagne, Merrill) que son demasiado pasivas, no pueden explotar el conocimiento anterior del alumno o utilizar errores como una oportunidad para aprender.

\subsection{Aprendizaje situado (Situated Learning)}

Inicialmente descrita por Jean Lave, la teoría del aprendizaje situado (Lave, 2003) se basa en el trabajo de Gibson (2003) y de Vygotsky (2003). Esta teoría (Lave \& Wenger, 1991) propone que el aprendizaje ocurre normalmente como función de una actividad, contexto o cultura; lo que se llama estar situada. La interacción social es crítica en el aprendizaje situado, los estudiantes se integran en una comunidad de prácticas que incorpora un conjunto de actitudes y comportamientos que los estudiantes van asumiendo paulatinamente.

Esta teoría dice que el conocimiento es una relación activa entre un agente y el entorno y el aprendizaje ocurre cuando el alumno está activamente envuelto en un contexto instruccional complejo y real (Young, 1993).

Gibson enfatiza que se aprende a través de la percepción y no de la memoria y sostiene que no solo el aprender sino también el pensar es situado y que por lo tanto debería ser considerado desde una perspectiva ecológica (Gibson, 1986).

Internet responde a las premisas del conocimiento situado en dos de sus características:

- Realismo: Posibilita intercambios auténticos entre usuarios provenientes de contextos culturales diferentes, pero con intereses similares.

- Complejidad: La naturaleza inestable del entorno en Internet constituye un escollo para los no iniciados (Brown, Collins \& Duguid, 1989). 


\section{3}

\section{EL NUEVO PARADIGMA DOCENTE}

\subsection{Diferentes modelos docentes}

Se repite en varios escritos (Troncoso-Saracho \& Alonso-Rodríguez, 1999) la afirmación según la cual la docencia no consiste en que el profesorado enseñe, sino en que el alumnado aprenda. Tortajada, Brusola \& Rubió (2009) define el modelo tradicional de docencia (Figura 2) como aquel cuyas características son:

- Primacía de los contenidos.

- Lección magistral como metodología predominante.

- Pasividad del alumnado.

- El profesorado es experto en contenidos y transmisor de información.

- La planificación es solo del profesorado.

- La única fuente de información son los apuntes de clase.

Un sistema de evaluación rígido basado exclusivamente en exámenes recopilatorios de conocimientos. 


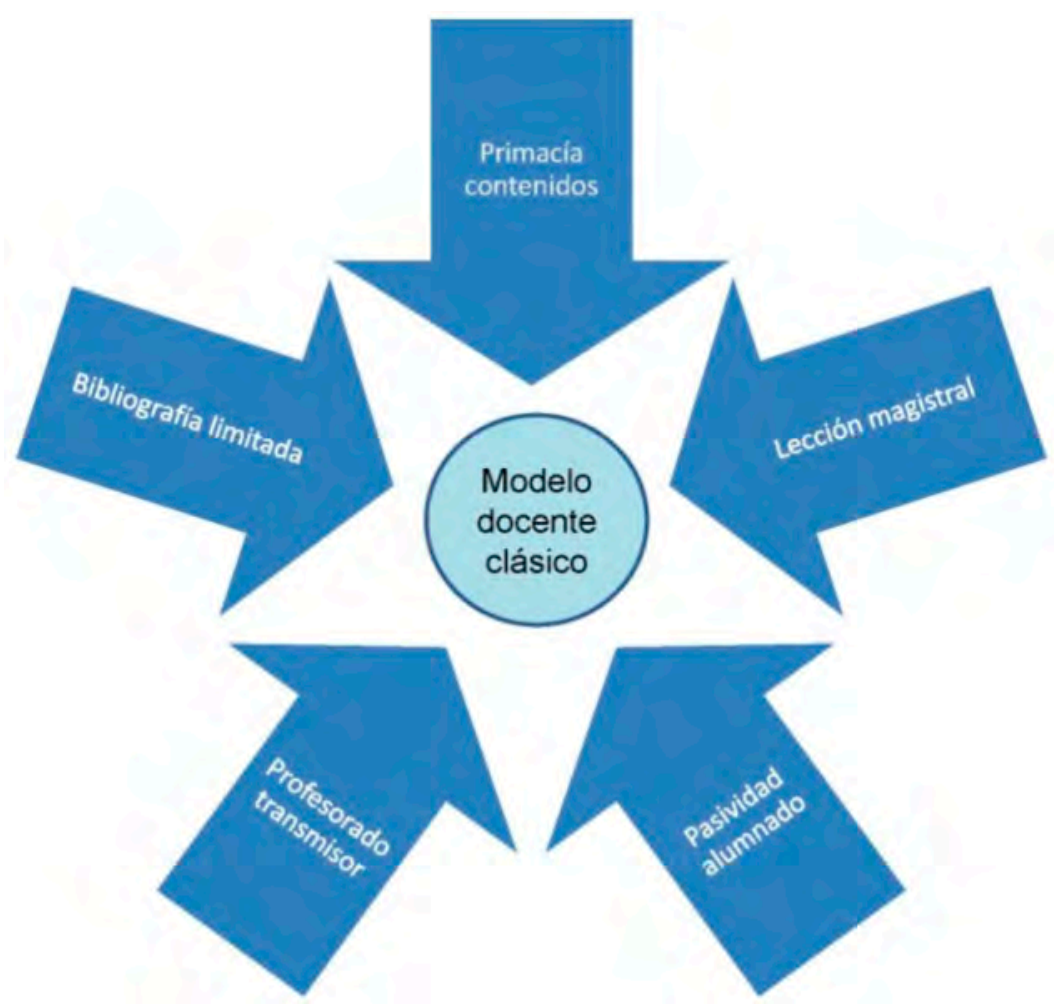

Figura 2. Modelo docente clásico.

Frente a este modelo, las nuevas tecnologías están cambiando significativamente la forma de enseñar; mientras que la docencia tradicional sigue métodos de trabajo artesanales es necesario proponer y replantear objetivos, contenidos y metodologías (Álvarez-Peñín, Charro-Hernández, García-Díaz \& Suarez-Quirós, 2000).

Existe la creencia de que la docencia ocupa un plano secundario en la actividad del docente dedicado fuertemente a la investigación. Por esto es necesaria una adaptación de la docencia a la realidad social. Se percibe que existe gran interés del profesorado en aprender y aplicar nuevos métodos que mejoren la calidad de la docencia; por ello es necesario que en los congresos especializados en docencia se presentan herramientas de apoyo para que este nuevo modelo de enseñanzaaprendizaje aporte un valor añadido a la docencia. Más que potenciar el punto de vista de innovación tecnológica, hay que utilizar esa tecnología para conseguir los objetivos de una forma mucho más eficaz (Sangrà, 2001). 
Para mejorar la docencia se proponen diferentes alternativas. Algunos autores (Herrero de Lucas, González-González, Acebes-Arconada, Fernando-Velázquez, Martín-Bravo, Mozo-Ruiz et al., 2008) destacan las ventajas de la coordinación entre el profesorado que imparten docencia en una misma titulación universitaria, tanto respecto a los contenidos, como respecto a las diferentes metodologías docentes utilizadas. Se propone coordinar la docencia en distintas áreas de conocimiento a fin de paliar la excesiva parcelación del conocimiento universitario. Para otros (Pallisera-Díaz \& Carretero-Torres, 2008) es imprescindible aportar a los docentes la información y formación suficiente para iniciar procesos innovadores en la docencia. Para otros (Bermúdez-Rodríguez, Lapaz-Castillo, MarquésCalvo, Morón-Tarifa, Povill-Cartoixà \& Voltas-Aguilar, 2000) las Tecnologías de la Información y la Comunicación (TIC) pueden suponer una potente ayuda a la docencia para mantener el nivel de calidad del área y adaptarse a las nuevas dedicaciones fijadas en los planes de estudio.

Parece claro que las TIC influirán de manera significativa en la docencia de manera que la conducirán inexorablemente a un nuevo cambio de paradigma. Las herramientas informáticas no solo plantean un cambio en la forma de aplicar la docencia sino también en los contenidos.

Otros autores (Marín-Granados, Gutiérrez-Ariza, García-Ceballos \& Mora-Segado, 2000) proponen un modelo de docencia en la que se combina la lección magistral, la tutorización y un sistema de enseñanza asistida por ordenador. La solución para aumentar la eficacia de la docencia, entendida como mayor cantidad de conocimiento impartido en menos tiempo, está en la animación asistida por ordenador, aunque destaca que este medio didáctico no puede utilizarse como único, ni de forma independiente al resto.

Martín-Gutiérrez, Martín-Dorta, Saorín-Pérez y Acosta-González (2006), identifica tres factores de cambio en la docencia: el nuevo modelo educativo diseñado por el Espacio Europeo de Educación Superior (EEES), la exigencia social de incorporación de las nuevas tecnologías de la información y comunicación a la universidad y la necesidad de mejorar la calidad universitaria.

Como soluciones para la mejora docente se propone:

- Mecanizar parte del trabajo de la docencia, sobretodo en cuanto a la gestión y corrección de ejercicios mediante nuevas herramientas basadas en las TIC (Álvarez-Peñín et al., 2000). 
- El uso de plataformas virtuales que permiten aumentar la calidad de la docencia, tanto a nivel de exigencia como de resultados (Zurita de la Vega \& TatoSánchez del Valle, 2008).

- La mejora de la eficiencia de la docencia mediante una propuesta metodológica basada en resultados obtenidos por el alumnado en los exámenes para conocer las causas que provocan el grado de dificultad (Metodología basada en el modelo de Rasch) (Rebollo-Castillo \& Álvarez-Martínez, 2009).

Es importante que el cambio del paradigma docente de la enseñanza al aprendizaje no suponga una merma en la calidad de la docencia. La figura 3 nos muestra el nuevo paradigma docente.

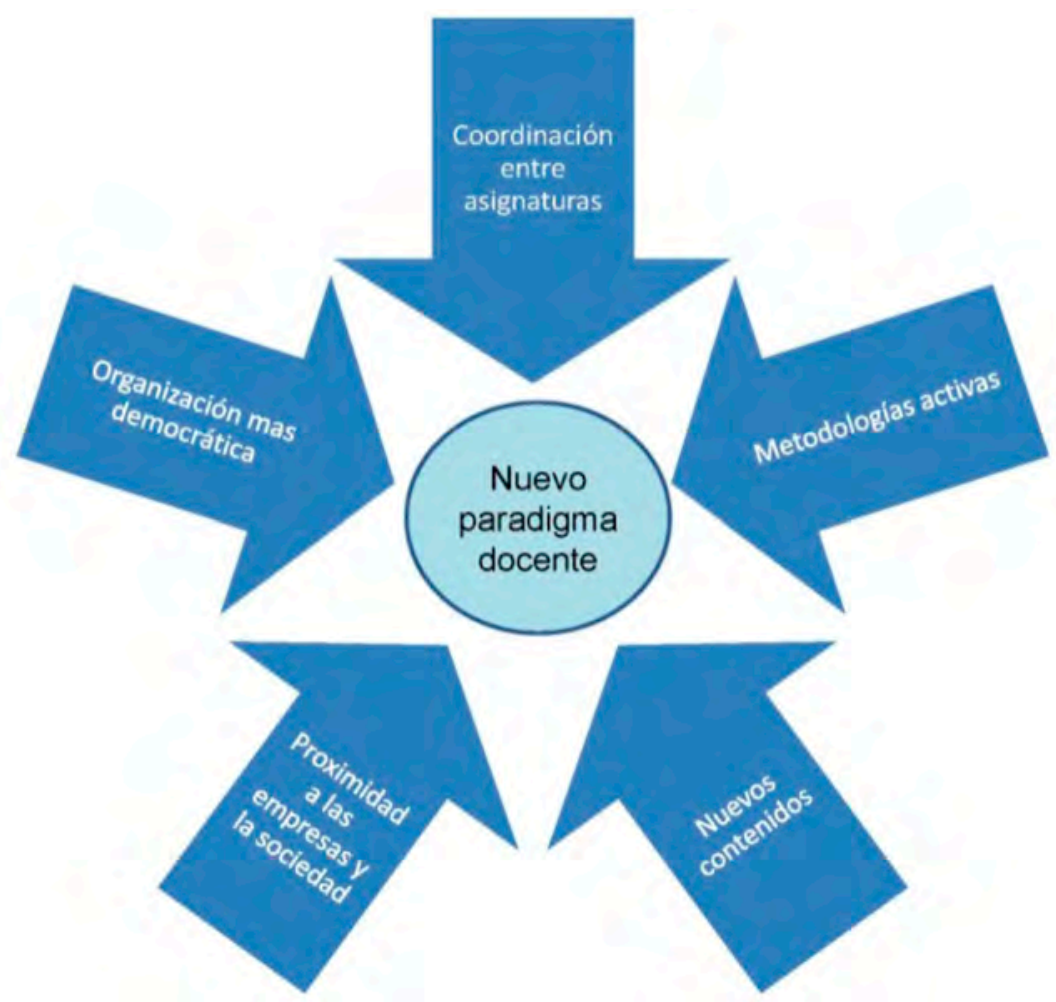

Figura 3. Nuevo paradigma docente. 


\subsection{Aprendizaje virtual}

Los orígenes del aprendizaje virtual (Pellejero-Salaberria, 2000) se basan en buena medida en el diseño de prácticas virtuales que permitían al alumnado desarrollar un tipo de aprendizaje de carácter interactivo. El aprendizaje virtual es el resultado de un proceso evolutivo histórico en el que se distinguen varias etapas: utilización de material impreso, docencia basada en material analógico, incorporación de herramientas informáticas y utilización de tecnología digital a través de Internet.

Las herramientas que facilitan el desarrollo de la realidad virtual han experimentado un gran avance, pues el equipo tecnológico necesario para ejecutar estas aplicaciones es simple y estándar, gracias en parte al nuevo escenario basado en la web, y ello permite una explosión de experiencias de aprendizaje virtual. Existe confianza de que, en el futuro, los laboratorios virtuales se convertirán en elementos integrados en los procesos de aprendizaje, incluso hay autores (Cruz, Blanco, Escalante \& Rivas, 2006) que afirman que el aprendizaje virtual facilitará la convergencia europea de los programas docentes universitarios. Una propuesta es incidir en el aprendizaje desde el lado del profesorado, mediante la creación de portales de aprendizaje virtuales temáticos de trabajo colaborativo de las distintas asignaturas.

Con la integración al EEES, algunas metodologías docentes sustentadas en la tecnología incorporan entornos virtuales de aprendizaje complementando las actividades presenciales, facilitando la publicación, la comunicación y el trabajo en grupo de manera que el modelo didáctico pasa a ser semipresencial. El nuevo entorno el aprendizaje virtual proporciona al alumnado la posibilidad de intervenir en su propio proceso de aprendizaje como un elemento de mejora docente. Para Zulueta-Pérez, Delgado-Urrecho \& Geijo-Barrientos (2009) toma cuerpo la idea de un aula virtual como una herramienta de apoyo a la docencia presencial, no como un recurso con el que elaborar conocimiento, sino como un vehículo de intercambio de información relevante y de mejora del aprendizaje.

Crear un entorno virtual tiene como objetivos:

- Introducir la asignatura en un entorno de trabajo basado en nuevas tecnologías. 
- Potenciar la planificación del trabajo de forma no presencial.

- Facilitar herramientas para la gestión de documentos.

- Fomentar el uso de las nuevas técnicas de integración ofimática y multimedia.

Las principales características de un aula virtual se pueden apreciar en la Figura 4.

Existe unanimidad (Moreno-Cazorla, 2000) en definir un aula virtual como un nuevo medio docente que supone grandes ventajas: flexibilidad de horario y lugar de la docencia, adaptación al ritmo de aprendizaje del alumnado, aprendizaje no lineal, e interactividad entre agentes docentes; pero también con sus limitaciones, sobretodo en temas de seguridad.

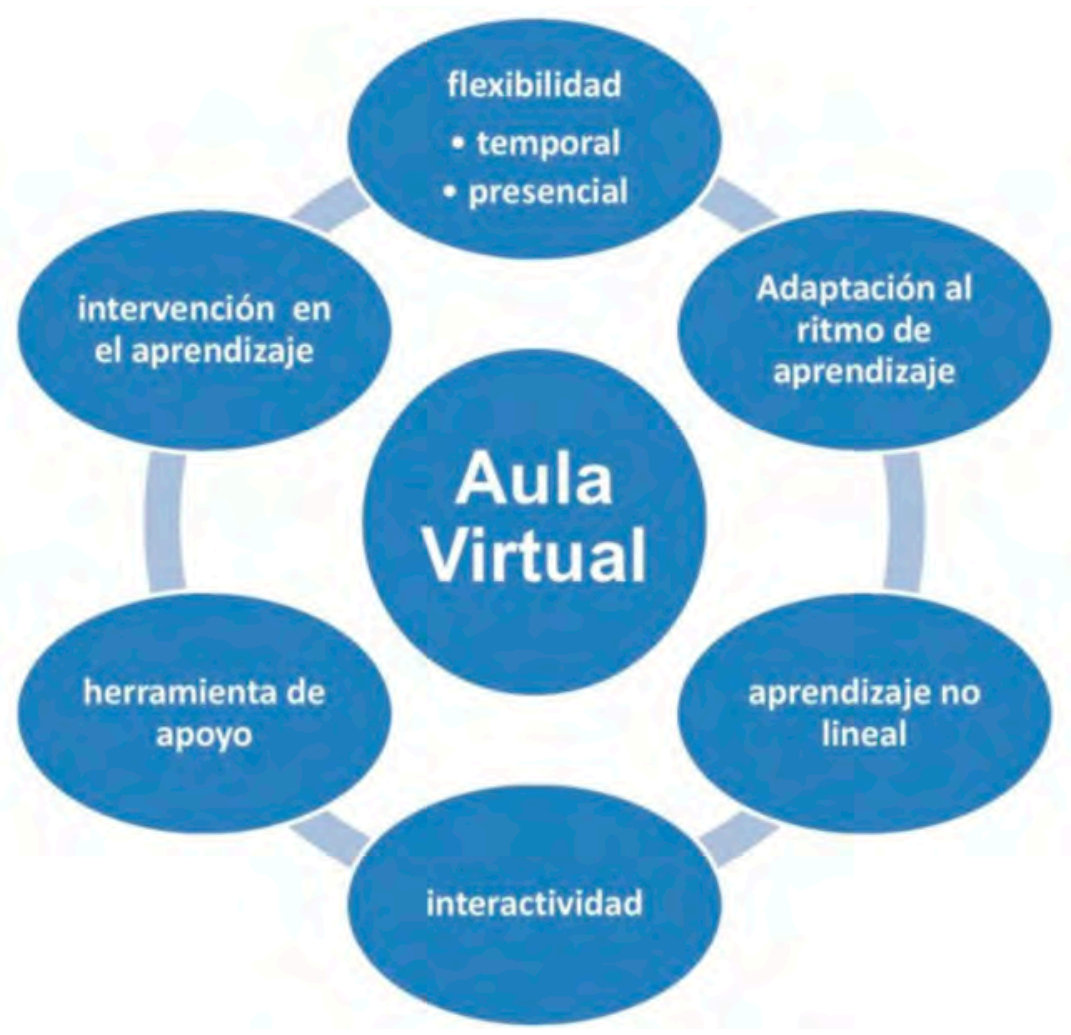

Figura 4. Características de un aula virtual. 
Hernández-Abad, Monguet-Fierro, Ocho-Vives, Hernández-Abad y Font-Andreu (2003), presenta dos tipos de entornos de aprendizaje virtuales: abiertos y cerrados. En general, se identifica a los entornos virtuales cerrados como sistemas que están bien planificados y que tienen una mayor eficiencia: el contenido de las materias ha sido creado y revisado por expertos, gran facilidad de uso, coherencia de los elementos que lo componen y donde el seguimiento del alumnado es una tarea compartida con un coordinador que asegura la uniformidad. Por el contrario, los sistemas abiertos, a pesar de no tener la eficiencia de los cerrados, destacan por: la planificación es más libre, la inversión en la generación del material es mucho menor, el material empleado es de más fácil preparación y hay una dependencia mucho mayor de la formación del profesorado y de su experiencia en la elaboración de material. La figura 5 resume lo expuesto.

Parece lógico pensar que las perspectivas de análisis y valoración de la enseñanza virtual puedan ser diferentes a la enseñanza presencial. La diferencia más significativa entre la educación en la presencialidad y en la virtualidad reside en el «cambio de medio». No podemos hacer lo mismo en dos medios diferentes. Aunque nuestra finalidad sea la misma (que los estudiantes comprendan lo que se les enseña), no podemos recorrer el mismo camino. El proceso de aprendizaje es un planteamiento global que se concreta de diferente forma en función del medio que se utiliza. Las clases virtuales se benefician de las posibilidades creativas de la red.

Según Sangrà (2001), los elementos diferenciales en los procesos educativos en entornos virtuales son de dos tipos: metodológicos y organizativos. Para este

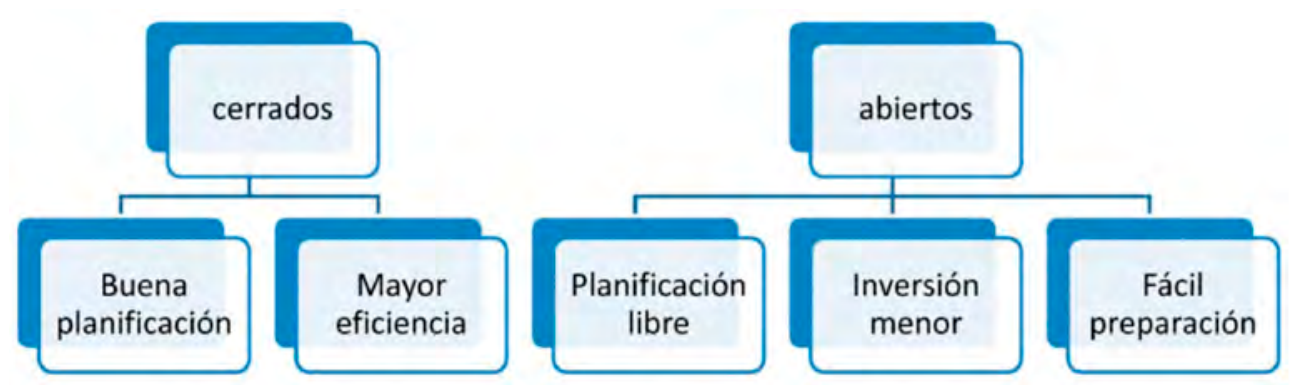

Figura 5. Entornos de aprendizaje virtual. 
autor, la metodología educativa para entornos virtuales de aprendizaje debe «estar centrada en el estudiante». Se trata de adaptar la metodología a un perfil de estudiante, con dificultades de tiempo para asistir a la universidad. Se trata de «llevar la universidad a casa de cada estudiante». Pero existen otros modelos en que la tecnología no cambia la docencia en sí, sino que tiene que ser un valor añadido a la tarea docente. Por otro lado, si en la docencia siempre tiene que existir un cierto entendimiento, en la docencia no presencial, el alumnado tiene que saber siempre qué se les va a exigir; esto supone una cierta negociación justo al empezar un curso de docencia no presencial. Para Gisbert (2002) existen dos ámbitos de aplicación claramente definidos, uno local (que funciona a través de un aula) y otro global (Internet).

Aprender con tecnología es diferente, implica al alumnado y le obliga a ser activo en el aprendizaje. La tecnología primero nos complica la vida (puesto que el aprendizaje es diferente para el estudiantado, pero también para el profesorado), pero con el tiempo se simplifica mucho más.

Gisbert propone organizar la docencia no presencial siguiendo una serie pautada de pasos:

- Planificar la asignatura.

- Diseñar y desarrollar la materia.

- Diseñar el proceso de implementación.

- Proponer una guía al alumnado.

- Planificar los diferentes bloques de contenidos.

- Desarrollar materiales de formación.

- Organizar mapas conceptuales.

- Crear materiales de evaluación.

La organización de la enseñanza en entornos virtuales debe poner al servicio del estudiante las posibilidades de las TIC. Las TIC no han de ser una finalidad 
en si misma sino tan solo un medio con un determinado valor añadido. En esta organización, los diferentes materiales de aprendizaje (debates, foros, mensajes, enlaces...) deben permitir la interacción y la construcción colectiva del conocimiento. La estructura particular en una organización virtual de educación ha de gestionar, además de los procesos académicos y docentes, la producción y edición de materiales educativos.

Pero... ¿Qué es la enseñanza virtual? ¿Es un nuevo paradigma, o sencillamente es la evolución de la enseñanza tradicional? Según Wedemeyer (1981), no existe un autentica teoría de la enseñanza virtual, aunque este autor se refiere a la tradicional enseñanza a distancia por correo convencional (Modelo UNED). Otros autores, por el contrario, sí que llegan a definir diferentes teorías propias para la educación a distancia: «La teoría de la autonomía e independencia del estudiante» (Delling, 1987), "La teoría basada en el proceso de industrialización de la educación» (Peters, 2002) y la «Teoría basada en la interacción y la comunicación» (Holmberg, 1987), pero fuere cual fuere la teoría estudiada, todas identifican tres elementos fundamentales para la enseñanza virtual:

- El estudiante (el tipo de estudiante específico de estos estudios).

- El docente (la relación que establece con el estudiante).

- Los recursos (permitentes de la interacción).

La relación que se establecen estos tres elementos en juego es lo que posibilita un nuevo paradigma en la docencia no presencial.

\subsection{Aprendizaje semipresencial}

Para Rajadell (2000), los estudios de carácter semipresencial se justifican en base a tres grandes ventajas:

1. Completar la oferta de estudios presenciales.

2. Ampliar el ámbito geográfico de los campus.

3. Permitir la compatibilidad de los estudios con las responsabilidades laborales. 
Para otros (Jiménez, Fernández, Pérez, Leo, Navarro, Arriza et al., 2008) su principal ventaja reside en que permite una introducción progresiva a la impartición a distancia, donde la semipresencialidad es solo un paso intermedio. En esta línea se manifiesta (Griful i Ponsati \& Gibert i Pedrosa, 2002) que expone que el cambio del modelo docente de presencial a semipresencial no tiene más motivación que la mejora de la calidad docente. También se destaca como elemento ventajoso (Lapaz-Castillo \& Voltas-Aguilar, 2002) que la semipresencialidad permite flexibilizar e individualizar el aprendizaje de los alumnos.

El éxito de la docencia semipresencial radica en conseguir que los estudiantes saquen el máximo provecho de las herramientas dispuestas para su aprendizaje, basándolo en un uso intensivo de les TIC. La utilización de entornos virtuales de aprendizaje en modalidad semipresencial se perfila como una estrategia de grandes posibilidades en la incorporación de las TIC, sobre todo en los estudios técnicos. Las herramientas que incorporan estos entornos virtuales permiten favorecer una metodología de aprendizaje basada en proyectos, en trabajo en grupo y en el asesoramiento personalizado. Los estudios semipresenciales siguen siempre el mismo Plan de Estudios que los presenciales, pero generalmente con una diferente distribución de las asignaturas entre obligatorias, optativas y libre elección, y alargando la carga de créditos en más semestres o cursos.

\subsection{Modelos de educación a distancia}

La educación a distancia existe desde muchos años antes de la aparición de Internet, pero con otros modelos docentes, puesto que ha sido una necesidad de la sociedad para poder satisfacer el aprendizaje de los ciudadanos que por razones de tiempo y/o espacio no podían asistir a la universidad pero tenían deseo de mejorar sus conocimientos. Taylor (2001) analizó cuatro modelos de educación a distancia que se han ido superando en el tiempo y en el espacio (existen países en que la educación a distancia esta en modelos más pretéritos que otros). Estos modelos están basados en la tecnología útil del momento histórico de su aplicación, lo que les ha permitido ciertas aplicaciones en unos casos y otras en los demás. Taylor destaca que justo cuando se está aplicando el cuarto modelo, está apareciendo un quinto, básicamente derivado del anterior, pero explotando todas las capacidades de Internet. Estos modelos son: 
- Primera generación-modelo basado en la correspondencia. La tecnología que usa es el papel. Este modelo ofrece flexibilidad en el tiempo y en el espacio. El alumno puede estudiar y hacer los ejercicios cuando quiere y donde quiere y luego enviarlos por correo. No existe interactividad.

- Segunda generación-modelo multimedia. Además del papel los soportes son el audio y el video. Este modelo ofrece flexibilidad en el tiempo y en el espacio. No existe interactividad.

- Tercera generación-modelo de teleaprendizaje. Usa la tecnología de la videoconferencia y las emisiones de televisión y radio. Este modelo ha perdido toda flexibilidad en tiempo y espacio puesto que hay que estar en un lugar determinado donde haya televisión para poder asistir a la emisión del programa que nos interesa y además a una hora determinada.

- Cuarta generación-modelo de aprendizaje flexible. Usa la tecnología multimedia interactiva on line e Internet. Este modelo ofrece flexibilidad en el tiempo y el espacio e interactividad. Suma las ventajas de la primera, segunda y tercera generación sin ninguna de sus limitaciones.

- Quinta generación-modelo de aprendizaje flexible inteligente. Usa la tecnología multimedia interactiva on line, Internet y campus virtual. Este modelo ofrece todas las ventajas de los anteriores y reduce significativamente el coste de la enseñanza.

No todos los autores están plenamente de acuerdo en estas cinco generaciones. Suárez-Quirós, García-Díaz, Álvarez-Peñín y Gallego-Santos (2002) reconoce sólo cuatro etapas en los modelos de educación a distancia: 1. Utilización de material impreso, 2. Docencia basada en material analógico, 3. Incorporación de herramientas informáticas y 4. Utilización de tecnología digital a través de Internet principalmente. De todas formas, el esquema es muy parecido, y en todos los casos se destaca que ha existido una evolución en la enseñanza a distancia siempre basada en la tecnología hábil en cada momento histórico, independientemente que existan cuatro o cinco modelos.

La aplicación de todo el potencial de las TIC en la enseñanza a distancia conlleva la aparición de un nuevo modelo de aprendizaje cada vez más eficiente y barato, con plena intercomunicación y que lleva aparejado un nuevo modelo de campus virtual, aunque se puede ir implementado en diferentes fases sucesivas. El 
potencial que Internet ofrece como herramienta docente se extiende a diversas perspectivas: Como complemento a la docencia presencial mediante el desarrollo de webs que contengan materiales de trabajo útiles al alumnado; como soporte a la gestión mediante herramientas que faciliten las tareas tanto docentes como administrativas y como aula virtual efectiva, llegando a crear en la red un infraestructura capaz de integrar todos los elementos docentes habitualmente empleados en la formación universitaria.

\subsection{Nuevos escenarios universitarios virtuales}

Se plantean dos posibles escenarios hacia donde podría evolucionar la organización de la enseñanza universitaria: el escenario local y el multinacional.

El escenario local, que se ha venido desarrollando desde los años 90 , consiste en un estallido del número de universidades cada vez más próximas físicamente al estudiante. Es un modelo a priori completamente ilógico con la explosión de las TIC. No hace falta llevar las universidades hasta la puerta de casa de cada estudiante puesto que las TIC permiten un aprendizaje a distancia. Pero en este escenario, lo que prima no es la tecnología existente sino la política, en la que cada región (e incluso ciudad) quiere «su» universidad.

El escenario global, al contrario, prevé cada vez menos universidades, puesto que el estudiante podrá escoger la universidad que mejor satisfaga sus necesidades independientemente de la distancia física del centro, merced a las TIC. De esta manera solo las «mejores» universidades podrán sobrevivir puesto que tendrán la suficiente masa crítica de estudiantes para poder sufragar los costos de la implementación de las nuevas tecnologías.

Pero existe un tercer escenario, no intermedio, sino mezcla de ambos. La proliferación de universidades en ciudades de poco peso demográfico, económico y/o tecnológico ha incentivado que estas instituciones desarrollasen todo el potencial de las TIC para poder atraer hacia ellas a estudiantes, no físicamente, sino a través de la red. De esta manera se lleva a cabo la implantación de una red de universidades (en general de la misma región) que comparten una serie de servicios. El ejemplo más paradigmático es el proyecto Intercampus, auspiciado por el Departament d'Universitats, Recerca i Societat de la Informació de la Generalitat de Catalunya. 
El proyecto Intercampus tiene como objetivo el intercambio de asignaturas de libre elección que se imparten en Internet. Las universidades participantes son: UAB, UB, UdG, UdL, UOC, UPC, UPF, y URV. Las universidades facilitan la comunicación y simplifican la burocracia, pero es un primer paso. A éste seguirán otros lógicos como el incremento de las asignaturas no solo las de libre elección, el compartimento de cada vez más información, y la expansión territorial (si la enseñanza está basada en las TIC, qué sentido tiene que el Intercampus sea de universidades catalanas y no puedan sumarse al proyecto otras instituciones españolas, latinoamericanas o de cualquier otro lugar del mundo). De este modo habremos llegado a un escenario global basado en universidades locales.

El proyecto Intercampus forma parte de hecho de un planteamiento mayor, la Universitat Digital de Catalunya, que pretende (además de conectar las asignaturas de libre elección no presenciales de las universidades catalanas):

- Crear una plataforma para producir y gestionar material educativo. Compartir materiales didácticos multimedia y sobre las metodologías profesor estudiante.

- Construir una plataforma en Internet de base a las editoriales de las universidades catalanas.

- Diseñar un servidor de tesis doctorales. Crear un consorcio virtual de universidades catalanas, el CBUC.

- Potenciar la conectividad universitaria catalana de Internet a nivel internacional, sobre todo con EEUU. 



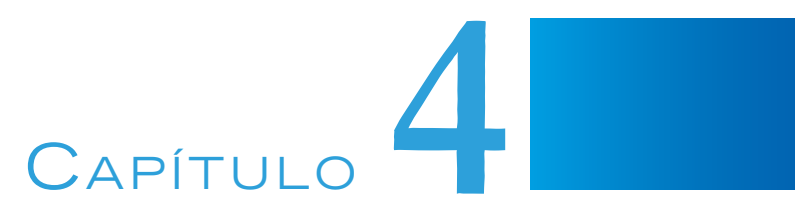

\section{CONCLUSIONES}

Las teorías del aprendizaje se agrupan en tres grandes modelos: Conductistas, Cognoscitivas y Constructivistas. El modelo constructivista es el más influyente en el ámbito de la didáctica de las ciencias de la educación y se reduce a cuatro submodelos principales tal y como se ha descrito en el presente libro, el constructivismo piagetiano, el humano, el movimiento de las concepciones alternativas (social) y el submodelo radical.

El constructivismo es parte esencial en el proceso de formación de los estudiantes, a la vez que la naturaleza de la interacción entre éste y la enseñanza es determinante en el aprendizaje. El procedimiento del aprendizaje es muchas veces de mayor importancia que el propio contenido o incluso de la forma en que la información es presentada.

La hipótesis constructivista es una de las teorías más influyentes en la educación, tanto en lo que respecta a las elaboraciones teóricas como por la propia práctica pedagógica. Los modelos constructivistas son útiles para diseñar estrategias de instrucción y técnicas que faciliten el aprendizaje, así como los fundamentos para seleccionarlos de manera eficaz.

Los modelos constructivistas parten de tres premisas básicas: La primera es que el alumno es el responsable de su propio aprendizaje, puesto que éste es un 
proceso activo de construcción más que de adquisición de conocimiento. La segunda idea fundamental destaca que los contenidos son el motor de la actividad de aprendizaje del alumno más que de comunicar conocimientos. Las dos ideas básicas anteriores no se aplican si el profesor no sabe crear las condiciones óptimas para el despliegue de actividades constructivistas, que es la tercera premisa necesaria para el éxito de los modelos constructivistas.

Las teorías de diseño instruccional pretenden analizar la forma en la que se estructura el aprendizaje, por lo que tienen gran importancia en la elaboración de materiales instruccionales. Los elementos de estas teorías se basan en una clasificación de los objetivos de aprendizaje, la prescripción de cómo descomponer los objetivos generales en específicos, la descripción de las acciones instructivas específicas, una prescripción de la secuencia de acciones instructivas definiendo la estrategia y un conjunto de condiciones para las acciones instructivas $\mathrm{v}$ las propias estrategias. 
Álvarez-Peñín, P, Charro-Hernández, M.E., García-Díaz, R.P., \& Suarez-Quirós, J. (2000). Análisis de la utilización de las NT en la ETSII de Gijón. Actas del VIII Congreso Universitario de Innovación Educativa en las Enseñanzas Técnicas. Donostia. ISBN 84-7585-402-8.

Argyris, C., \& Schon, D.A. (1974). Theory in practice: Increasing professional effectiveness. Jossey-Bass.

Argyro, R. (2003). CSCL: Computer Supported Collaborative Learning.

Ausubel, D.P. (1963). La psicología del aprendizaje verbal significativo. Una introducción al aprendizaje escolar. Nueva York/Londres.

Ausubel, D.P. (1982). Psicología educativa. Un punto de vista cognoscitivo. México: Trillas.

Aguirre, M., Meza, S., \& Lucero, I. (2005). La potencialidad de la V de Gowin en la resolución de problemas. Facultad de Ciencia Exactas y Naturales y Agrimensura. Corrientes, Argentina.

Bandura, A. (2005) Teoría Cognitiva Social del Aprendizaje.

Bandura, A., Adams, N.E., \& Beyer, J. (1977). Cognitive processes mediating behavioral change. Journal of Personality and Social Psychology, 35(3), 125-139. https://doi.org/10.1037/0022-3514.35.3.125

Barrows, H. \& Tamblyn, R. (1980). Problem based learning: An approach to medical education. NY: Springer. 
Bartlett, F.C. (1932). Remembering: An experimental and social study. Cambridge: Cambridge University.

Bermúdez-Rodríguez, F., Lapaz-Castillo, J.L., Marqués-Calvo, J., Morón-Tarifa, M., Povill-Cartoixà, D., \& Voltas-Aguilar, J. (2000). Utilización de las nuevas tecnologías de la información y de las comunicaciones en las asignaturas de carácter semi-presencial. XII INGEGRAF. Valladolid.

Bodner, G.M. (1986). Constructivism: A theory of knowledge. Journal of Chemical Education, 63(10, 873.

Bransford, J.D., Sherwood, R.D., Hasselbring, T.S., Kinzer, C.K., \& Williams, S.M. (1990). Anchored instruction: Why we need it and how technology can help. En Nix, D., \& Spiro, R.J. (Eds.). Cognition, education, and multimedia: Exploring ideas in high technology (115-141). Hillsdale, NJ: Lawrence Erlbaum Associates.

Brown, J.S., Collins, A., \& Duguid, S. (1989). Situated cognition and the culture of learning. Educational Researcher, 18(1), 32-42.

Bruner, J.S. (1966). Toward a theory of instruction. Cambridge: Harvard University Press.

Card, S., Moran, T., \& Newell, A. (1983). The Psychology of Human-Computer Interaction. Hillsdale, NJ: Erlbaum.

Carretero, M. (1993). Constructivismo y educación. Buenos Aires. 1993.

Carretero, M. (1998). Desarrollo y aprendizaje. Buenos Aires. 1998.

Carroll, J.M. (1998). Minimalism beyond the Nurnberg Funnel. Cambridge, MA: MIT Press.

Carroll, J.M., \& Rosson, M.B. (1987). Paradox of the active user. The MIT Press.

Casas, A., Fíalo, L., \& Maia, L.J. (2003). La tercera generación de la enseñanza. Ambientes inteligentes para la educación basados en realidad virtual.

Castro-Pereira, M. (2004). ¿Es la andragogía una ciencia? 
Charney, D.H., Reder, L.M., \& Wells, G.W. (1988). Studies of elaboration of instructional texts. En Doheny-Farina, S. (Ed). Effective Documentation: What Have We Learned From Research (47-72). Cambridge, MA: MIT Press.

Coll, C. (1990). Aprendizaje Escolary Construcción del Conocimiento. Paidós.

Coll, C. (1997). Constructivismo y educación escolar: ni hablamos siempre de lo mismo ni lo hacemos siempre desde la misma perspectiva epistemológica. En Rodrigo, M.J. \& Arnay J. (Comps.). La construcción del conocimiento escolar. Barcelona: Paidós.

Coll, C. (1998). La teoría genética y los procesos de construcción del conocimiento en el aula. En Castorina, J.A., Coll, C. \& otros: Piaget en la educación. Debate en torno a sus aportaciones. Buenos Aires: Paidós.

Cronbach, L. \& Snow, R. (2003). Aptitude-Treatment Interaction.

Cross, K.P. (1976). Accent on Learning. San Francisco: Jossey-Bass.

Cross, K.P. (1981). Adults as Learners. San Francisco: Jossey-Bass.

Cruz, A., Blanco, A., Escalante, M., \& Rivas, A. (2006). Resultados de una experiencia de aprendizaje virtual en alumnos universitarios. III CIDUI. Girona.

Delling, R.M. (1987). Towards a theory of distance education. ICDE Bulletin, 13, 21-25.

Dewey, J. (1916). Method in science teaching. Columbia University. DOI: https:/ / doi. org/10.1002/sce.3730010101

Driver, R., \& Easley, J. (1978). Pupils and paradigms: A review of literature related to concept development in adolescent science students. Studies in Science Education, 5, 61-84. https://doi.org/10.1080/03057267808559857

Duffy, T.M., \& Jonassen, D.H. (1991). Constructivism: New implication for instructional technology. Educational Technology, 31(5), 7-12.

Duffy, T.M., \& Jonassen, D.H. (1992). Constructivism and the technology of instruction: A conversation. Hillsdale, NJ: Lawrence Erlbaum Publishers. 
Duffy, T.M., \& Cunningham, D.J. (1996). Constructivism: implications for the design and delivery of instruction. En Jonassen, D.H. (Ed.). Handbook of research for educational communications and technology. A Project of the Associations for Educational Communications and Technology. New York, USA: Macmillan Library Reference USA.

Duffy, T.M.; Lowyck, J., \& Jonassen, D.H. (1992). The design of constructivistic learning environments: Implications for instructional design and the use of technology. Heidelburg, Germany: Springer-Verlag.

Ehrenfels, C.V. (1890). Über gestaltqualitäten. Vierteljahrsschrift für Wissenschaftliche Philosophie, 14(3), 249-292.

Fernández, A. (2003). Coordinador Técnico de la Unidad de Virtualización Académica de la UNED.

Gagne, R.M., \& Dick, W. (1983). Instructional Psychology. En Rosenzweig, M., \& Porter, 1. (Eds.). Annual Review of Psychology. Palo Alto, CA: Annual Reviews. https://doi.org/10.1146/annurev.ps.34.020183.001401

Gardner, H., \& Hatch, T. (1989). Educational implications of the theory of multiple intelligences. Educational Researcher, 18(8), 4-10.

Gisbert, M. (2002). Docència i noves tecnologies: Recomanacions Pedagògiques. Ponencia en jornadas Las TIC en la docencia en la UPC: experiencias realizadas. UPC.

Gibson, J.J. (1986). The ecological approach to visual perception. Hillsdale, NJ: Lawrence Erlbaurn.

Gibson, J.J. (2003). Information Pickup Theory.

Glaser, R. (1982). Instructional psychology: Past, Present, Future. American Psychology, 37(3), 292-305.

Gómez-Granell, C., \& Coll, C. (1994). De qué hablamos cuando hablamos de constructivismo, Cuadernos de Pedagogía, 221, 8-10. 
Griful i Ponsati, E., \& Gibert i Pedrosa, J. (2002). Un Segon Cicle semipresencial en Escola Tècnica Superior Enginyeria Industrial de Terrassa. II CIDUI. Tarragona.

Hackos, J.T., \& Redish, J. (1998). User and task analysis for interface design. Wiley.

Hartman, F.R. (1961). Investigation of recognition learning under multiple-chanel presentation and testing conditions. Av Communication Review, 9, 24-43.

Hernández-Abad, F., Monguet-Fierro, J.M., Ocho-Vives, M., HernándezAbad, V., \& Font-Andreu, J. (2003). Infraestructura y espacios virtuales para el desarrollo de la enseñanza a distancia. XIII ADM - XV INGEGRAF. Italia.

Herrero de Lucas, L.C., González-González, M.L., Acebes-Arconada, L.F., Fernando-Velázquez, M., Martín-Bravo, M.Á., Mozo-Ruiz, I. et al. (2008). Hacia una nueva realidad docente: adaptación al EEES de la titulación de ingeniería técnica industrial, en la Escuela Universitaria Politécnica de Valladolid. V CIDUI. Lleida.

Holmberg, B. (1987). Research Review: The development of distance education research. American Journal of Distance Education, 1(3), 16-23.

Hull, C.L (1920). Quantitative aspects of the evolution of concepts. Psychological Monographs, 28(1).

Jacoby, B., \& Associates (1996). Service-Learning in Higher Education: Concepts and Practices. San Francisco: Jossey-Bass.

Jáuregui, K. (2002). Formación a través de la tecnología en la literatura. IESE.

Jiménez, F., Fernández, C., Pérez, F., Leo, T.J., Navarro, E., Arriza, P. et al. (2008). Rediseño de asignaturas para su impartición en formato semipresencial o a distancia. XVI CUIEET. Cádiz.

Jiménez, M.P. (1991). Análisis de modelos didácticos: Didáctica de las Ciencias. Módulo I, Curso de Formación del Profesorado de Ciencias, MEC. 
Jonassen, D.H. (1991). Objectivism versus Constructivism: Do we need a new Philosophical paradigm? Educational Research Technology \& Development, 39, 5-14.

Jonassen, D.H. (2000). El diseño de entornos constructivistas de aprendizaje. En Reigeluth C.M. (Ed.). Diseño de la instrucción. Teorías y modelos (225-250). Madrid: Santillana.

Jonassen, D.H. (2005). Tecnología del pensamiento: Hacia un modelo de diseño constructivista.

Jonassen, D.H., \& Reeves, T. (1996). Learning with technology: Using Computers as Cognitive Tools. En Jonassen, D.H. (Ed.). Handbook of research for educational communications and technology. A Project of the Associations for Educational Communications and Technology. New York, USA: Macmillan Library Reference USA.

Jonassen, D.H., Peck, K., \& Wilson, B. (1999) Learning with technology: A constructivist perspective. Merrill.

Kahn, P.H., \& Friedman, B. (1993). Control and power in educational computing. Paper presented at the Annual Meeting of the American Educational Research Association.

Kaynama, S.A., \& Keesling, G. (2000). Development of a Web-based Internet marketing course. Journal of Marketing Education, 22(2), 84-89.

Kearsley, G., \& Shneiderman, B. (1997). Engagement Theory: A framework for technology-based teaching and learning. The Virtual Professor: A Personal Case Study.

Kemp, J.E., \& Smellie, D.C. (1989). Planning, producing, and using instructional media. New York: Harper and Row Publishers.

Kelly, E.L. (1955). Consistency of the adult personality. American Psychologist, 10(11), 659.

Kieras, D.E. (1988). Towards a practical GOMS model methodology for user interface design. En Helander, M. (Ed.) Handbook of Human-Computer Interaction. Amsterdam: Elsevier/North Holland. 
Knowles, M. (1968). Andragogy, not pedagogy. Adult Leadership, 16(10), 350-352.

Knowles, M. (1980). The modern practice of adult education: from pedagogy to andragogy. Englewood Cliffs: Prentice Hall/Cambridge.

Knowles, M. (1984). Andragogy in action: Applying modern principles of adult education. San Francisco: Jossey-Bass.

Kozma, R.B. (1991). Learning with media. Review of educational research, 61, 179-211.

Lapaz-Castillo, J.L., \& Voltas-Aguilar, J. (2002). Nuevos enfoques en la docencia del CAD y la normalización eléctrica. XIV INGEGRAF. Santander.

Lave, J. (2003). Teoría del conocimiento situado.

Lave, J., \& Wenger, E. (1991). Situated Learning: Legitimate Peripheral Participation. Cambridge, UK: Cambridge University Press. https://doi.org/10.1017/ CBO9780511815355

Lazonder, A.W., \& van der Meij, H. (1995). Error-information in tutorial documentation: supporting users' errors to facilitate initial skill learning. International Journal of Human-Computer Studies, 42(2), 185-206.

Lewin, K, \& Gullickson, T. (1948). Resolving Social Conflicts: Field Theory in Social Science. Psyccritiques. 42(8), 756. https://doi.org/10.1037/005017

Londoño, F.C. (2005). Interfaces de las Comunidades Virtuales. Manizales: Universidad de Caldas.

Makkonen, P. (1998). Learning of Basic Concepts in Informatics Using Collaborative Hypertext: Does Collaborative Hypertext Support Learning as a Whole?

Martín-Gutiérrez, J., Martín-Dorta, N., Saorín-Pérez, J.L., \& Acosta-González, M. (2006). Campus virtual en la docencia de expresión gráfica en la ingeniería en la universidad de La Laguna. XVIII INGEGRAF. Barcelona.

Marín-Martínez, N. (2003). Visión constructivista dinámica para la enseñanza de las ciencias. Departamento de Didáctica de la Matemática y de las Ciencias Experimentales. Universidad de Almería. 
Marín-Granados, M.D., Gutiérrez-Ariza, F.J., García-Ceballos, M.L., \& MoraSegado, P. (2000). La Enseñanza Asistida por Ordenador como recurso eficaz en el proceso enseñanza-aprendizaje. XII INGEGRAF. Valladolid.

Marín-Martínez, N., Solano-Martínez, I., \& Jiménez-Gómez, E. (1999). Tirando del hilo de la madeja constructivista. Enseñanza de las Ciencias: Revista de Investigación y Experiencias Didácticas 17(3), 479-492.

Martín, E. (1992). La fundamentación psicológica del currículum de la Reforma educativa. Ed. Educación Abierta. Instituto de Ciencias de la Educación de la Universidad de Zaragoza.

Mergel, B. (2005). Diseño instruccionaly teoría del aprendizaje.

Merrill, M.D. (1983). Component Display Theory. En Reigeluth, C. (Ed.). Instructional Design Theories and Models. Hillsdale, NJ: Erlbaum Associates.

Merrill, M.D. (1994). Instructional Design Theory. Englewood Cliffs, NJ: Educational Technology Publications.

Merrill, M.D. (1999). Instructional Transaction Theory (ITT): Instructional Design Based on Knowledge Objects. En Reigeluth, C.M. (Ed.) InstructionalDesign Theories and Models: A New Paradigm of Instructional Theory. Mahwah, NJ: Lawrence Erlbaum Associates.

Miller, G. (1956). The magical number seven, plus or minute two: Some limits on our capacity for processing information. Psychological Review, 63, 81-97.

Moore, D.M., Burton, J.K., \& Myers, R.J. (1996). Multiple-Channel Communication: The Theoretical and Research Foundations of Multimedia. Handbook of research for educational communications and technology. A Project of the Associations for Educational Communications and Technology. New York, USA: Macmillan Library Reference USA.

Moreira, M.A., \& Novak, J.D. (1988). Investigación en enseñanza de las ciencias en la Universidad de Cornell: esquemas teóricos, cuestiones centrales y abordes metodológicos. Enseñanza de las ciencias: revista de investigación y experiencias didácticas, 6(1), 3-18. 
Moreno-Cazorla, R. (2000). Nuevas tecnologías de la información y las comunicaciones en el ámbito docente de la Expresión Gráfica: El CAD como punto de partida y no como disciplina complementaria. XII INGEGRAF. Valladolid.

Nieda, J., \& Macedo, B.(2005). Las fuentes del currículo. OEI.

Novak, J.D. (1988). Constructivismo humano: un consenso emergente. Enseñanza de las Ciencias: Revista de Investigación y Experiencias Didácticas, 6(3), 213-223.

Novak, J.D. (1997). Teoría y práctica de la educación (Vol. 330). Spain: Anaya-Spain.

Paivio, A. (1986). Mental representations: A dual coding approach. Nueva York: Oxford University Press.

Pallisera-Díaz, M., \& Carretero-Torres, R. (2008). Implicación de la Facultad de Educación y Psicología en la mejora de la docencia y la reforma de los planes de estudios. V CIDUI. Lleida.

Pavlov, I.P (1927). Conditioned reflexes: an investigation of fhe physiological activity of the cerebral cortex. Oxford University

Pellejero-Salaberria, I. (2000). Sistema Multimedia aplicado a la mejora del proceso enseñanza-aprendizaje en el estudio de las Bombas Hidráulicas. VIII CUIEET. Donostia. ISBN 84-7585-402-8.

Papert, S., \& Harel, I. (2002). Situar el construccionismo. Alajuela: INCAE.

Peters, O. (2002). La educación a distancia en transición. Nuevas tendencias y retos. Universidad de Guadalajara.

Piaget, J. (1954). Les relations entre l'intelligence et l'affectivité dans le développement de l'enfant. Paris: Centre de Documentation Universitaire.

Piaget, J. (1981). Infancia y Aprendizaje. Journal for the Study of Education and Development, $4(\sup 2)$. 
Rajadell-Carreras, M., \& Astals-Coma, F. (2000). Las enseñanzas semipresenciales: la experiencia de la ETSI de Terrassa. VIII CUIEET. Donostia. ISBN 84-7585-402-8.

Rebollo-Castillo, F.J., \& Álvarez-Martínez, P. (2009). Desarrollo de una propuesta metodológica para evaluar la docencia de la asignatura Sistemas de Información Geográfica basada en el modelo de Rasch. XXI INGEGRAF - XVII $A D M$. Lugo.

Reigeluth, C.M. (1999). Instructional Design Theories and Models. A New Paradigm of Instructional Theory. Mahwah, NJ: Lawrence Erlbaum Associates.

Resnick, L. (1999). La educación y el aprendizaje del pensamiento. Buenos Aires: Aique.

Revans, R. (1998). ABC of Action Learning: Empowering managers to act and to learn from action. Lemons \& Crane.

Rouet, J.F., Levonen, J J., Dillon, A.P., \& Spiro, R.J. (Eds.) (1996). Studying and Learning with hypertext. Empirical Studies and theirs Implications. En Hipertext and cognitions. Mahwah, NJ: Lawrence Erlbaum Associates.

Saba, F. (1988). Integrated systems of telecommunications and the transaction instructional. The American Journal of Distance Education, 2(3).

Sagales, P. (2001). Universidad Nacional de Asunción.

Sangrà, A. (2001). Enseñar y aprender en la virtualidad. Revista Educar UAB, 28, ISSN0211-819-X.

Santacruz-Valencia, L. (2000). Elo: entorno para la generación, integración y reutilización de objetos de aprendizaje. Universidad Carlos III. Madrid.

Schank, R.C. (1999). Learning by doing. En Reigeluth (Ed.). Instructional Design Theories and Models. A New Paradigm of Instructional Theory (Vol II) (161-181). Mahwah, NJ: Lawrence Erlbaum Associates. https://doi.org/10.1017/ CBO9780511527920.011 
Schank, R.C. (2001). The Computer isn't the Medium, It's the Message. Communications of the ACM, 44(3), 142-143.

Schön, D.A. (1987). Educating the reflective practitioner: Toward a new design for teaching and learning in the professions. Jossey-Bass.

Schunk, D.H. (1999). Learning Theories: An Educational Perspectives. Pearson.

Shneiderman, B. (1994). Education by Engagement and Construction: Can Distance Education be Better than Face-to-Face?

Simon, H. (1916). Models of a man. Cambridge. Massachusetts: The MIT'T Press.

Skinner, B.F (1938). The Behavior of organisms. B.F. Skinner Foundation. ISBN 978-0-9964539-0-5.

Streibel, M.J. (1991). Instructional Design and Human Practice: What Can We Learn from Habermas' Theory of Technical and Practical Human Interests?

Suárez-Quirós, J., García-Díaz, R.P., Álvarez-Peñín, P.I., \& Gallego-Santos, R. (2002). Nuevas tecnologías en la enseñanza virtual a través de la red. XIV INGEGRAF. Santander.

Taylor, J.C. (2001). The future of learning-learning for the future: Shaping the transition. Open Praxis, 2, 20-24.

Tejada, J. (1999). Manual impreso minimalista versus manual hipermedia: Contraste empírico de dos tipos de materiales de adiestramiento informático para usuarios inexpertos. Revista electrónica LEEME, 4.

Thorndike, E.L (1906). The principles of teaching: Based on psychology. Routledge. ISBN 0415-21012-7.

Tolman, E.C., Hall, C.S., \& Bretnall, E.P. (1932). A disproof of the law of effect and a substitution of the laws of emphasis, motivation and disruption. Journal of Experimental Psychology, 15(6), 601-614. https://doi.org/10.1037/ h0073609 
Tortajada, I., Brusola, F. \& Rubió, C. (2009). Objetos de aprendizaje aplicados a la asignatura de gestión de los procesos de color. XVII CUIEET. Valencia.

Troncoso-Saracho, J., \& Alonso-Rodríguez, J.A. (1999). Medios didácticos aplicables en la enseñanza del Dibujo. VII CUIEET. Huelva.

Van der Meij, H. (1995). Principles and heuristics for designing minimalist instruction. Technical communication, 42(2), 243-261.

Viennot, O. (1976). Temples de l'inde centrale et occidentale: Planches. École Française d'Extrême-Orient.

Vygotsky, L.S. (1978). Mind in Societ: Development of Higher Psychological Processes. Harvard University Press.

Vygotsky, L.S. (2003). Social Development Theory.

Watson, J.B (1916). The place ot the conditioned-reflex in psychology. Psychological Review, 23, 89-116.

Wedemeyer, C. (1981). Learning at the Back-door. Madison: University of Wisconsin.

Wertsch, J.V. (1988). Vygotsky y la formación social de la mente. Paidós.

Wiley, D.A. (2000). Connecting learning objects to instructional design theory: A definition, a metaphor, and a taxonomy. Digital Learning Environments Research Group. II Utah State University. The Edumetrics Institute. Emma Eccles Jones Education 227.

Winn, W. (1990). Some implications of cognitive theory for instructional design. Instructional Science, 19(1), 53-69.

Winn, W. (1993). A conceptual basis for educational applications of virtual reality. Technical Publication R-93-9. Human Interface Technology Laboratory of the Washington Technology Center, Seattle: University of Washington.

Young, M.F. (1993). Instructional design for situated learning. Educational Technology Research \& Development, 41(1), 43-58. 
Zulueta-Pérez, P., Delgado-Urrecho, J., \& Geijo-Barrientos, J.M. (2009). Análisis de los recursos y actividades propios de la plataforma virtual Moodle para su utilización como apoyo a la docencia presencial de expresión gráfica en la ingeniería. XXI INGEGRAF-XVII ADM. Lugo.

Zurita de la Vega, E., \& Tato-Sánchez del Valle, P. (2008). Formación a través de plataformas virtuales. XX INGEGRAF. Valencia. 



\section{AUTORES}

Noelia Olmedo Torre es doctora Cum Laude en Ingeniería Multimedia por la Universidad Politécnica de Catalunya (UPC-BarcelonaTech) y es Ingeniera de Telecomunicaciones. Tiene dos Posgrados: Diseño de Sistemas Interactivos Multimedia y Enseñanza Universitaria en Ciencias, Tecnología, Ingeniería y Matemáticas - STEM. Es profesora del Departamento de Expresión Gráfica en la Ingeniería (EGE) en la Escola d'Enginyeria de Barcelona Est (EEBE). Imparte las asignaturas de Proyectos de Ingeniería y Expresión Gráfica. Trabajó en el Laboratorio Multimedia (LAM) de la UPC coordinando diferentes proyectos de e-learning. Es miembro de dos grupos de investigación: INSIDE (Innovació en Sistemes per al Disseny i la Formació a l'Enginyeria) y GIIP (Grup de Recerca en Enginyeria de Projectes: Disseny, Sostenibilitat i Comunicació). Su investigación se centra en aspectos sociales de la educación en la ingeniería, competencias profesionales, herramientas de evaluación, promoción y mejora de la enseñanza y el aprendizaje y, en general, la innovación educativa. Ha publicado más de 30 trabajos científicos en este campo. Desde 2015 forma parte del equipo editorial de la revista JOTSE (Journal of Technology and Science Education). Desde 2017 es Secretaria Académica del Instituto de Ciencias de la Educación (ICE-UPC). 
Oscar Farrerons Vidal es arquitecto por la Escola Tècnica Superior d'Arquitectura de Barcelona (ETSAB) y doctor Cum Laude en Ingeniería Multimedia por la Universitat Politècnica de Catalunya (UPC). Posee el posgrado Enseñanza Universitaria en Ciencias, Tecnología, Ingeniería y Matemáticas (STEM). Desde 1995 es profesor del Consorci Escola Industrial de Barcelona, y desde 2000 de la Escola Universitària d'Enginyeria Tècnica Industrial de Barcelona. Actualmente es profesor de la Escola d'Enginyeria de Barcelona Est (EEBE). Ha ejercido enseñanza universitaria en Proyectos y desde el año 2000 en el departamento de Expresión Gráfica en la Ingeniería. Ha desplegado diferentes asignaturas de ingeniería gráfica durante más de veinte años en la UPC, y ha sido coordinador de la asignatura troncal «Expressió Gràfica». Desde 2017 es subdirector del Departamento de Expresión Gráfica en la Ingeniería de la UPC. Forma parte de los grupos de investigación «Enginyeria de Projectes» (GIIP) y «Innovació en Sistemes per al Disseny i la Formació a l'Enginyeria» (INSIDE). Posee más de treinta años de experiencia profesional en diseño gráfico de ingeniería civil como colaborador en despachos profesionales de Barcelona. Ha llevado a cabo auditorías técnicas de proyectos de ingeniería. Sus ámbitos de colaboración profesional están circunscritos a ingeniería civil, urbanismo, sostenibilidad en ingeniería, accesibilidad, e investigación de aguas naturales. Miembro de la Mesa Territorial de Adaptación al Cambio Climático del Montseny (MéTACC) en el proyecto LIFE-CLINOMICS. 
Con más de 20 años de docencia universitaria en carreras técnicas a sus espaldas, los autores de este libro vienen observando desde hace tiempo cómo el constructivismo es parte esencial en el proceso de formación de los estudiantes y como la naturaleza de la interacción entre éste y la instrucción es determinante en el aprendizaje, siendo de igual o mayor importancia que el contenido o la forma en que la información es presentada.

Los autores realizan su actividad docente muy implicados con los modelos GOMS, Learning by Doing y Situated Learning, además de Problem Based Learning y el Método del Caso, que los han llevado a alcanzar altas cotas de rendimiento entre su alumnado y que el lector puede descubrir a través de las numerosas publicaciones realizadas en revistas de prestigio.

El libro que tiene en sus manos realiza una revisión de las teorías y modelos más importantes del constructivismo e intenta dar luz al gran abanico de propuestas metodológicas en la consecución y el desarrollo de una enseñanza de mayor calidad.
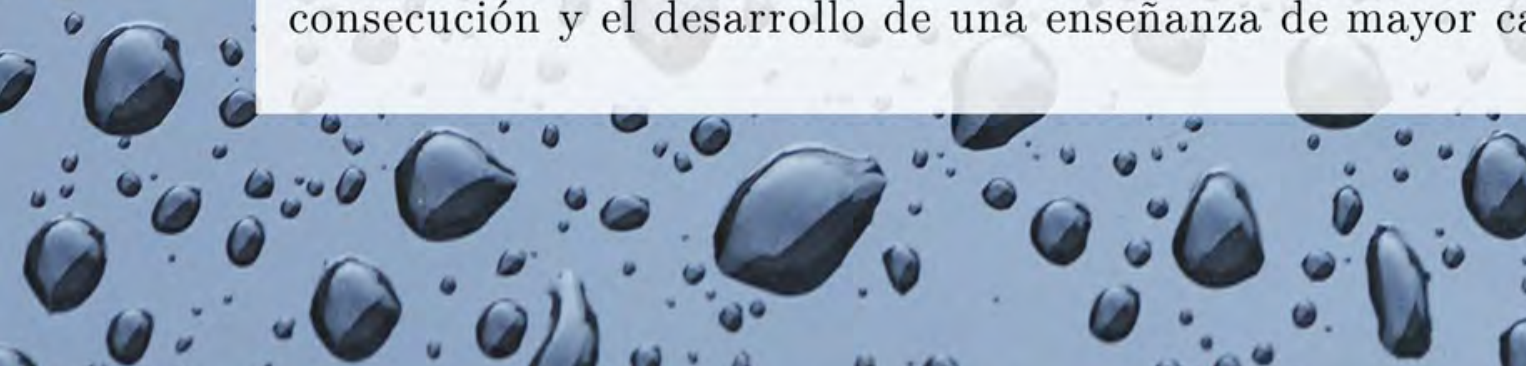